\title{
Refocused continuous-wave decoupling: A new approach to heteronuclear dipolar decoupling in solid-state NMR spectroscopy
}

\author{
Joachim M. Vinther, ${ }^{1}$ Anders B. Nielsen, ${ }^{1}$ a) Morten Bjerring, ${ }^{1}$ Ernst R. H. van Eck, ${ }^{2}$ \\ Arno P. M. Kentgens, ${ }^{2}$ Navin Khaneja, ${ }^{1,3, b)}$ and Niels Chr. Nielsen ${ }^{1, b)}$ \\ ${ }^{1}$ Center for Insoluble Protein Structures (inSPIN), Interdisciplinary Nanoscience Center (iNANO) and \\ Department of Chemistry, Aarhus University, Gustav Wieds Vej 14, DK-8000 Aarhus C, Denmark \\ ${ }^{2}$ Physical Chemistry/Solid-State NMR, Institute for Molecules and Materials, Radboud University Nijmegen, \\ P.O. Box 9010, 6500 GL Nijmegen, The Netherlands \\ ${ }^{3}$ Division of Applied Sciences, Harvard University, Cambridge, Massachusetts 02138, USA
}

(Received 24 August 2012; accepted 12 November 2012; published online 6 December 2012)

\begin{abstract}
A novel strategy for heteronuclear dipolar decoupling in magic-angle spinning solid-state nuclear magnetic resonance (NMR) spectroscopy is presented, which eliminates residual static high-order terms in the effective Hamiltonian originating from interactions between oscillating dipolar and anisotropic shielding tensors. The method, called refocused continuous-wave $(r \mathrm{CW})$ decoupling, is systematically established by interleaving continuous wave decoupling with appropriately inserted rotor-synchronized high-power $\pi$ refocusing pulses of alternating phases. The effect of the refocusing pulses in eliminating residual effects from dipolar coupling in heteronuclear spin systems is rationalized by effective Hamiltonian calculations to third order. In some variants the $\pi$ pulse refocusing is supplemented by insertion of rotor-synchronized $\pi / 2$ purging pulses to further reduce the residual dipolar coupling effects. Five different $r \mathrm{CW}$ decoupling sequences are presented and their performance is compared to state-of-the-art decoupling methods. The $r \mathrm{CW}$ decoupling sequences benefit from extreme broadbandedness, tolerance towards rf inhomogeneity, and improved potential for decoupling at relatively low average rf field strengths. In numerical simulations, the $r \mathrm{CW}$ schemes clearly reveal superior characteristics relative to the best decoupling schemes presented so far, which we to some extent also are capable of demonstrating experimentally. A major advantage of the $r \mathrm{CW}$ decoupling methods is that they are easy to set up and optimize experimentally. (C) 2012 American Institute of Physics. [http://dx.doi.org/10.1063/1.4768953]
\end{abstract}

\section{INTRODUCTION}

Heteronuclear decoupling is a prerequisite for solid-state nuclear magnetic resonance (NMR) spectroscopy of organic and biological systems with abundant ${ }^{1} \mathrm{H}$ spins and detection of low- $\gamma$ nuclei such as ${ }^{13} \mathrm{C}$ and ${ }^{15} \mathrm{~N}$. The mere fact that the decoupling performance to a large extent translates directly into the resolution in the solid-state NMR spectra has rendered the development of efficient decoupling methods a hot topic in solid-state NMR for years. A large variety of methods have been proposed that significantly improve the performance of standard continuous wave $(\mathrm{CW})$ decoupling with inspiration taken from composite pulses, ${ }^{1-3}$ symmetry-based recoupling such as $\mathrm{C} 7,{ }^{4} \mathrm{CN}$, and $\mathrm{RN}$ sequences, ${ }^{5}$ as well as numerical and experimental optimizations. ${ }^{6}$ Decoupling sequences such as TPPM (two pulse phase modulation), ${ }^{7}$ SPINAL (small phase incremental alternation), ${ }^{8} \mathrm{XiX}$ (Xinverse-X), ${ }^{9}$ PISSARRO (phase-inverted supercycled sequence for attenuation of rotary resonance), ${ }^{10} \mathrm{CN}$ - and $\mathrm{RN}$ decoupling, ${ }^{11}$ and eDROOPY (experimental decoupling is robust for offset or power inhomogeneity $)^{6}$ have markedly improved heteronuclear decoupling in solid-state NMR. Lately,

\footnotetext{
a) Present address: Physical Chemistry, ETH Zürich, Wolfgang-Pauli-Strasse 10, 8093 Zürich, Switzerland.

b) Authors to whom correspondence should be addressed. Electronic addresses: navin@seas.harvard.edu and ncn@inano.au.dk.
}

attention has been devoted to improving the broadbandedness of the decoupling through frequency sweeping and supercycling, leading to decoupling sequences such as swept TPPM $\left(\mathrm{SW}_{f}\right.$-TPPM), ${ }^{12,13}$ swept SPINAL (SW ${ }_{f}$-SPINAL), ${ }^{14}$ and supercycled-swept TPPM. ${ }^{15}$ From comparative studies, it is evident that the optimal decoupling sequence to a large extent depends on the experimental conditions, including in particular the magic-angle-spinning (MAS) frequency and the applicable decoupling rf field strength. ${ }^{16}$ These two parameters are of considerable practical importance as both sample spinning and high-power rf irradiation may cause substantial sample heating, unless special E-field free probes ${ }^{17-20}$ or extensive sample cooling (which may cause temperature gradients in the sample) are used. Obviously, issues like sensitivity also come into play in this discussion as large sample volumes obviously will be restricted to rotors not capable of ultrafast spinning and often associated with reduced decoupling capabilities relative to that available for the smallest rotor/rf coil diameters.

Obtaining high-resolution solid-state NMR spectra relies on many facts in addition to the decoupling sequence, including local order in the sample (as explored through production of nano- or microcrystals and highly order fibers ${ }^{21-24}$ ), nuclear spin relaxation properties, ${ }^{25}$ and multiple-spin effects. ${ }^{26}$ While the former is an intrinsic property of the sample that only can be altered through sample preparation, the two 
latter may be influenced through the use of fast sample spinning serving to reduce, e.g., ${ }^{1} \mathrm{H}-{ }^{1} \mathrm{H}$ interactions. ${ }^{27,28}$ Adapting a reverse strategy, recoupling of homonuclear protonproton couplings may be used as a means to decouple residual heteronuclear dipolar couplings and exploit this as an additional decoupling field or source to so-called selfdecoupling. ${ }^{11,29}$ Obviously, both relaxation and multiple-spin effects may also be altered through isotope labeling, e.g., using extensive deuteration. ${ }^{30,31}$

In this paper, we address heteronuclear decoupling for ${ }^{13} \mathrm{C}$ (or ${ }^{15} \mathrm{~N}$ ) NMR spectroscopy in fully protonated samples and return to the simplest setup of isolated $\mathrm{CH}$ - and a $\mathrm{CH}_{2}$-spin systems to explore in more detail the possibility to improve the decoupling performance through systematic removal of residual heteronuclear dipolar coupling terms. Our entry is a third-order effective (or average) Hamiltonian analysis $^{32-34}$ of standard CW decoupling experiments, revealing dipolar coupling and dipolar-coupling-involved cross terms which, in principle, straightforwardly may be canceled by simple modifications in the decoupling sequence. Major focus is devoted to improving tolerance towards resonance offsets, chemical shift anisotropy, and rf inhomogeneity, while simultaneously ensuring easy establishment of robust decoupling conditions experimentally. These aims lead to the concept of refocused-CW $(r \mathrm{CW})$ decoupling sequences which amounts to standard CW decoupling with appropriate insertion of strong refocusing pulses (potentially supplemented with purging pulses) in synchrony with the sample spinning. Overall, we present five different $r \mathrm{CW}$ decoupling sequences which illustrate our design principle. All sequences are rationalized theoretically, numerically, and experimentally with reference to state-of-the-art recoupling methods.

\section{EFFECTIVE HAMILTONIAN ANALYSIS}

In relation to solid-state NMR, a heteronuclear IS twospin-1/2 system (e.g., $I$ and $S$ representing ${ }^{1} \mathrm{H}$ and ${ }^{13} \mathrm{C}$, respectively) may be characterized by the following Hamiltonians: the isotropic and anisotropic chemical shift for the proton $H_{I}^{C S}$, the isotropic and anisotropic chemical shift for the carbon $H_{S}^{C S}$, and the scalar and dipolar couplings involving the two spins $H_{I S}^{J}$ and $H_{I S}^{D}$. The scalar coupling is assumed decoupled completely by the $\mathrm{CW}$ decoupling ( $J$ couplings much smaller than the applied rf field amplitude) and is therefore not included in the following analysis. For an IJS three-spin$1 / 2$ system resembling a $\mathrm{CH}_{2}$-group, the chemical shift of the extra proton (represented by $J$ ) $H_{J}^{C S}$, and the two dipolar couplings $H_{J S}^{D}$ and $H_{I J}^{D}$ have to be included as well.

In the high-field approximation and under MAS conditions, the individual Hamiltonians may conveniently be described as

$$
H^{\lambda}=\omega_{\lambda}(t) T^{\lambda}
$$

where the irreducible spherical tensor operator $T^{\lambda}$ describes spin-field components of the nuclear spin interaction $\lambda$. To first order this operator takes the form $B_{0} I_{z}, B_{0} S_{z}, \frac{2}{3} B_{0} I_{z}$, $\frac{2}{3} B_{0} S_{z}, \frac{1}{\sqrt{6}} 2 I_{z} S_{z}$, and $\frac{1}{\sqrt{6}}\left(2 I_{z} J_{z}-I_{x} J_{x}-I_{y} J_{y}\right)$ for the $I-$ spin and $S$-spin isotropic and anisotropic chemical shift, the heteronuclear dipole-dipole coupling, and the homonuclear dipole-dipole coupling interactions, respectively. The instantaneous amplitude (in angular frequency units) for a given interaction $\lambda$ may conveniently be cast in terms of a Fourier series

$$
\omega_{\lambda}(t)=\sum_{m=-2}^{2} \omega_{\lambda}^{(m)} e^{i m \omega_{r} t}
$$

with $\omega_{r} / 2 \pi$ denoting the spinning frequency in $\mathrm{Hz}$. The Fourier coefficients are generally given as

$$
\omega_{\lambda}^{(m)}=C^{\lambda} \sum_{m^{\prime}=-2}^{2}\left(R_{2, m^{\prime}}^{\lambda}\right)^{P} D_{m^{\prime},-m}^{(2)}\left(\Omega_{P R}^{\lambda}\right) d_{-m, 0}^{(2)}\left(\beta_{R L}\right),
$$

where $C^{\lambda}$ and $\left(R_{2, m^{\prime}}^{\lambda}\right)^{P}$ represent the fundamental constant and the principal-axis-frame $(P)$ spatial tensor for the interaction $\lambda$, respectively. $D_{m^{\prime},-m}^{(2)}\left(\Omega_{P R}^{\lambda}\right)$ is a Wigner rotation matrix element with $\Omega_{P R}^{\lambda}$ being the Euler angles describing the orientation of the principal-axis-frame interaction tensor relative to the rotor frame $(R) . d_{-m, 0}^{(2)}\left(\beta_{R L}\right)$ is a reduced Wigner rotation matrix element with $\beta_{R L} \sim 54.74^{\circ}$ (MAS). For the axially symmetric dipole-dipole coupling interaction under MAS conditions this leads to the non-vanishing terms

$$
\begin{aligned}
& \omega_{D}^{(1)}=\omega_{D}^{(-1) *}=\frac{1}{2 \sqrt{2}} b_{I S} \sin \left(2 \beta_{P R}\right) e^{i \gamma_{P R}}, \\
& \omega_{D}^{(2)}=\omega_{D}^{(-2) *}=-\frac{1}{4} b_{I S} \sin ^{2}\left(\beta_{P R}\right) e^{i 2 \gamma_{P R}},
\end{aligned}
$$

with $b_{I S}=-\left(\gamma_{I} \gamma_{S} / r_{I S}^{3}\right) \frac{\mu_{0}}{4 \pi}$ being the dipole-dipole coupling constant depending on the internuclear distance $r_{I S}$ and the gyromagnetic ratios $\gamma_{I}$ and $\gamma_{S}$ of the two spins. Under the same conditions, the chemical shift is described by the Fourier components

$$
\begin{aligned}
\omega_{C S}^{(m)}= & \omega_{\text {iso }} \delta_{m, 0}+\omega_{\text {aniso }}\left(D_{0,-m}^{(2)}\left(\Omega_{P R}^{C S}\right)\right. \\
& \left.-\frac{\eta_{\text {aniso }}}{\sqrt{6}}\left(D_{-2,-m}^{(2)}\left(\Omega_{P R}^{C S}\right)+D_{2,-m}^{(2)}\left(\Omega_{P R}^{C S}\right)\right)\right) \\
& \times d_{-m, 0}^{(2)}\left(\beta_{R L}\right),
\end{aligned}
$$

with $\omega_{\text {iso }}$ being the isotropic chemical shift, $\delta_{m, 0}$ a Kronecker delta, $\omega_{\text {aniso }}$ the chemical shift anisotropy (CSA), and $\eta_{\text {aniso }}$ the chemical shift tensor asymmetry parameter. The Euler angles denoted $P R$ represent the transformation from the principal axis $(P)$ to the rotor $(\mathrm{R})$ frame, while those marked $R L$ represent the transformation from the rotor to the laboratory frame $(L)$ (under MAS conditions, we have $\beta_{R L}$ $=\arccos (1 / \sqrt{3}))$.

Upon transformation into the interaction frame of an $I$ spin $x$-phase CW rf irradiation field $\left(H_{r f}=\omega_{r f} I_{x}\right)$, the relevant Hamiltonians for the $I S$ spin system take the form

$$
\begin{gathered}
\tilde{H}_{S}^{C S}=\omega_{S}(t) S_{z}, \\
\tilde{H}_{I}^{C S}=\omega_{I}(t)\left(\cos \left(\omega_{r f} t\right) I_{z}+\sin \left(\omega_{r f} t\right) I_{y}\right), \\
\tilde{H}_{I S}^{D}=\omega_{D}(t)\left(\cos \left(\omega_{r f} t\right) 2 I_{z} S_{z}+\sin \left(\omega_{r f} t\right) 2 I_{y} S_{z}\right),
\end{gathered}
$$


which for the $I$-spin chemical shift and $I S$ dipole coupling may be reformulated to

$$
\begin{aligned}
\tilde{H}_{I}^{C S}= & \sum_{m=-2}^{2} \omega_{I}^{(m)}\left(\frac{1}{2}\left(e^{i\left(m \omega_{r}+\omega_{r f}\right) t}+e^{i\left(m \omega_{r}-\omega_{r f}\right) t}\right) I_{z}\right. \\
& \left.+\frac{1}{2 i}\left(e^{i\left(m \omega_{r}+\omega_{r f}\right) t}-e^{i\left(m \omega_{r}-\omega_{r f}\right) t}\right) I_{y}\right) \\
\tilde{H}_{I S}^{D}= & \sum_{m=-2}^{2} \omega_{D}^{(m)}\left(\frac{1}{2}\left(e^{i\left(m \omega_{r}+\omega_{r f}\right) t}+e^{i\left(m \omega_{r}-\omega_{r f}\right) t}\right) 2 I_{z} S_{z}\right. \\
& \left.+\frac{1}{2 i}\left(e^{i\left(m \omega_{r}+\omega_{r f}\right) t}-e^{i\left(m \omega_{r}-\omega_{r f}\right) t}\right) 2 I_{y} S_{z}\right) .
\end{aligned}
$$

The relevant effective Hamiltonians (involving individual interactions or cross terms between different interactions) up to third order is calculated using the standard formulae $\mathrm{e}^{32}$

$$
\begin{gathered}
\overline{\tilde{H}}^{(1)}=\frac{1}{\tau_{c}} \int_{0}^{\tau_{c}} d t \tilde{H}(t) \\
\overline{\tilde{H}}^{(2)}=\frac{1}{2 i \tau_{c}} \int_{0}^{\tau_{c}} d t_{2} \int_{0}^{t_{2}} d t_{1}\left[\tilde{H}\left(t_{2}\right), \tilde{H}\left(t_{1}\right)\right] \\
\overline{\tilde{H}}^{(3)}=\frac{-1}{6 \tau_{c}} \int_{0}^{\tau_{c}} d t_{3} \int_{0}^{t_{3}} d t_{2} \int_{0}^{t_{2}} d t_{1}\left(\left[\tilde{H}\left(t_{3}\right),\left[\tilde{H}\left(t_{2}\right), \tilde{H}\left(t_{1}\right)\right]\right]\right. \\
\left.+\left[\tilde{H}\left(t_{1}\right),\left[\tilde{H}\left(t_{2}\right), \tilde{H}\left(t_{3}\right)\right]\right]\right) .
\end{gathered}
$$

Here the cycle time, denoted $\tau_{c}$, is set to $2 \pi p / \omega_{r}$ which ensures periodicity of $H_{r f}$ for a commensurate rf amplitude and spinning frequency $p \omega_{r f}=q \omega_{r}$, with $p, q$ being integers. It is noted that convergence of the effective Hamiltonian is only warranted for $p$ and $q$ having a small common multiple. In the following we use the notation $n=q / p$, leading to $\omega_{r f}=n \omega_{r}$.

To second order, under the same conditions, the only contribution influencing the $S$-spin involves a cross term between the $I$-spin chemical shift interaction and the heteronuclear dipolar coupling

$$
\overline{\tilde{H}}^{(2)}=-2 I_{x} S_{z} \sum_{n=1}^{2}\left(\left(\omega_{I}^{(-n)} \omega_{D}^{(n)}+\omega_{I}^{(n)} \omega_{D}^{(-n)}\right) \frac{\omega_{r f}}{n^{2} \omega_{r}^{2}-\omega_{r f}^{2}}\right),
$$

being identical to the result previously obtained by Ernst et $a .^{35}$ using Floquet theory.
The second-order term, however, does not explain the observed spin evolution sufficiently well (vide infra), implying that higher-order terms in the effective Hamiltonian need to be evaluated. To third order, the only non-vanishing terms are a direct term from the dipole-dipole coupling and a cross term between the $I$-spin chemical shift anisotropy and the heteronuclear dipole-dipole coupling. In the derivation of this term, we initially evaluate the relevant commutators of $H^{(3)}$.

As a start, we define the Hamiltonian (cf. Eqs. (8) and (9))

$$
\begin{aligned}
\tilde{H}(t) & =\tilde{H}_{I}^{C S}(t)+\tilde{H}_{I S}^{D}(t) \\
& =\left(\omega_{I}(t)+\omega_{D}(t) 2 S_{z}\right)\left(\cos \left(\omega_{r f} t\right) I_{z}+\sin \left(\omega_{r f} t\right) I_{y}\right) \\
& =\left(\omega_{I}(t)+\omega_{D}(t) 2 S_{z}\right) \tilde{I}_{z}(t) .
\end{aligned}
$$

Now, keeping only terms involving the $S$-spin, the commutators of $\overline{\tilde{H}}^{(3)}$ in Eq. (14) can be evaluated to

$$
\begin{aligned}
& {\left[\tilde{H}\left(t_{3}\right),\left[\tilde{H}\left(t_{2}\right), \tilde{H}\left(t_{1}\right)\right]\right]+\left[\tilde{H}\left(t_{1}\right),\left[\tilde{H}\left(t_{2}\right), \tilde{H}\left(t_{3}\right)\right]\right]} \\
& =\left(\left[\tilde{I}_{z}\left(t_{3}\right),\left[\tilde{I}_{z}\left(t_{2}\right), \tilde{I}_{z}\left(t_{1}\right)\right]\right]+\left[\tilde{I}_{z}\left(t_{1}\right),\left[\tilde{I}_{z}\left(t_{2}\right), \tilde{I}_{z}\left(t_{3}\right)\right]\right]\right) \\
& \quad \times 2 S_{z}\left(\omega_{D}\left(t_{3}\right) \omega_{D}\left(t_{2}\right) \omega_{D}\left(t_{1}\right)+\omega_{D}\left(t_{3}\right) \omega_{I}\left(t_{2}\right) \omega_{I}\left(t_{1}\right)\right. \\
& \left.\quad+\omega_{I}\left(t_{3}\right) \omega_{D}\left(t_{2}\right) \omega_{I}\left(t_{1}\right)+\omega_{I}\left(t_{3}\right) \omega_{I}\left(t_{2}\right) \omega_{D}\left(t_{1}\right)\right),
\end{aligned}
$$

using the definition $\tilde{I}_{z}(t)=\cos \left(\omega_{r f} t\right) I_{z}+\sin \left(\omega_{r f} t\right) I_{y}$. Comparing with the result obtained only taking the dipolar coupling into account, i.e.,

$$
\begin{aligned}
{\left[\tilde{H}_{I S}^{D}\left(t_{3}\right),\left[\tilde{H}_{I S}^{D}\left(t_{2}\right), \tilde{H}_{I S}^{D}\left(t_{1}\right)\right]\right] } & \\
+ & {\left[\tilde{H}_{I S}^{D}\left(t_{1}\right),\left[\tilde{H}_{I S}^{D}\left(t_{2}\right), \tilde{H}_{I S}^{D}\left(t_{3}\right)\right]\right] } \\
= & \left(\left[\tilde{I}_{z}\left(t_{3}\right),\left[\tilde{I}_{z}\left(t_{2}\right), \tilde{I}_{z}\left(t_{1}\right)\right]\right]+\left[\tilde{I}_{z}\left(t_{1}\right),\left[\tilde{I}_{z}\left(t_{2}\right), \tilde{I}_{z}\left(t_{3}\right)\right]\right]\right) \\
& \times 2 S_{z}\left(\omega_{D}\left(t_{3}\right) \omega_{D}\left(t_{2}\right) \omega_{D}\left(t_{1}\right)\right)
\end{aligned}
$$

one obtains a great simplification of the calculations of the third-order cross term, since the contribution from the $I$-spin chemical shift anisotropy only adds three terms to the expression. The commutators in Eqs. (16) and (17) evaluate to a sum of triple cosine and sine products that may be recast in the form of $e^{ \pm i \omega_{r f} t_{1}} e^{ \pm i \omega_{r f} t_{2}} e^{ \pm i \omega_{r f} t_{3}}$ and the integral of the thirdorder effective Hamiltonian can be evaluated for each term using the triple integrals given in the supplementary material. ${ }^{36}$ Under the assumption $\omega_{r f} \neq n \omega_{r}$ for $n=0, \pm 1, \pm 2, \pm 3, \pm 4$, \pm 5 , \pm 6 , the third-order effective Hamiltonian becomes

$$
\begin{aligned}
\overline{\tilde{H}}^{(3)}= & \frac{1}{12} \sum_{m=-2}^{2} \sum_{n=-2}^{2} \frac{1}{m^{2} n^{2} \omega_{r}^{4}-\left(m^{2}+n^{2}\right) \omega_{r}^{2} \omega_{r f}^{2}+\omega_{r f}^{4}} \\
& \times\left(2 I _ { z } S _ { z } ( m n \omega _ { r } ^ { 2 } + 3 \omega _ { r f } ^ { 2 } ) \left\{\omega_{D}^{(m)}\left(\omega_{D}^{(-m)}+\omega_{D}^{(-n)}\right) \omega_{D}^{(n)}+\omega_{I}^{(m)}\left(\omega_{I}^{(-m)}+\omega_{I}^{(-n)}\right) \omega_{D}^{(n)}\right.\right. \\
& \left.+\omega_{I}^{(m)}\left(\omega_{D}^{(-m)}+\omega_{D}^{(-n)}\right) \omega_{I}^{(n)}+\omega_{D}^{(m)}\left(\omega_{I}^{(-m)}+\omega_{I}^{(-n)}\right) \omega_{I}^{(n)}\right\} \\
& +2 I_{y} S_{z} i \omega_{r} \omega_{r f}\left\{\omega_{D}^{(m)}\left((m+3 n) \omega_{D}^{(-m)}+(3 m+n) \omega_{D}^{(-n)}\right) \omega_{D}^{(n)}+\omega_{I}^{(m)}\left((m+3 n) \omega_{I}^{(-m)}+(3 m+n) \omega_{I}^{(-n)}\right) \omega_{D}^{(n)}\right. \\
& \left.\left.+\omega_{I}^{(m)}\left((m+3 n) \omega_{D}^{(-m)}+(3 m+n) \omega_{D}^{(-n)}\right) \omega_{I}^{(n)}+\omega_{D}^{(m)}\left((m+3 n) \omega_{I}^{(-m)}+(3 m+n) \omega_{I}^{(-n)}\right) \omega_{I}^{(n)}\right\}\right) .
\end{aligned}
$$


It is noted that we have also derived both the secondand the third-order terms using Peano-Baker series ${ }^{37-39}$ and an anisotropic (jolting) frame transformation ${ }^{40}$ which serves to confirm our results.

Both the second- and the third-order terms are seen to depend on the strength of the heteronuclear dipolar coupling and the proton chemical shift anisotropy. While the secondorder term depends on the product of the dipolar coupling and the proton CSA, the third-order term constitutes a sum of the dipolar coupling to power 3 and the square of the proton CSA multiplied by the dipolar coupling. From this analysis, it becomes clear that a large dipolar coupling non-surprisingly leads to large residual (unwanted) terms, but also that a large proton CSA (being particularly important under high-field conditions) may contribute considerably to the residual line broadening. Considering typical values for the ${ }^{1} \mathrm{H}-{ }^{13} \mathrm{C}$ dipoledipole coupling and proton CSA's in proteins being $b_{I S} / 2 \pi$ $\sim 23 \mathrm{kHz}$ and $\delta_{\text {aniso }}^{C S} \sim-3.5 \mathrm{ppm}$, then even for very high static magnetic fields ( $1 \mathrm{GHz}{ }^{1} \mathrm{H}$ resonance frequency) and CW decoupling with rf amplitude $100 \mathrm{kHz}$, the third-order term involving only the dipolar coupling is of the same order of magnitude as the second-order term. This underlines the importance of the third-order term in the effective Hamiltonian. Since, under high-power $\mathrm{CW}$ irradiation conditions, the third-order terms scale with $\omega_{r f}^{-2}$ (for $I_{z} S_{z}$ ) and $\omega_{r f}^{-3}$ (for $I_{y} S_{z}$ ) while the second-order term scales with $\omega_{r f}^{-1}$, the relative importance of the third-order term obviously decreases with increasing rf field strength. We should note, however, that the proton CSA can be as large as $10-15 \mathrm{ppm},{ }^{41,42}$ implying that in high-field applications one should not underestimate the role of the cross terms involving the ${ }^{1} \mathrm{H}$ CSA.

For well-specified small spin systems, it is possible to calculate the contributions from the second- and third-order effective Hamiltonian terms. Figure 1 compares the absolutevalue averages over the powder angles $\beta_{P R}$ and $\gamma_{P R}$ of the amplitudes for the second- and third-order terms proportional to $I_{x} S_{z}, I_{z} S_{z}$, and $I_{y} S_{z}$ for a $\mathrm{CH}$ spin system (see Sec. IV for details) under conditions of a ${ }^{1} \mathrm{H}$ resonance frequency of $850 \mathrm{MHz}$ and $20 \mathrm{kHz}$ MAS. It is indeed the case that the third-

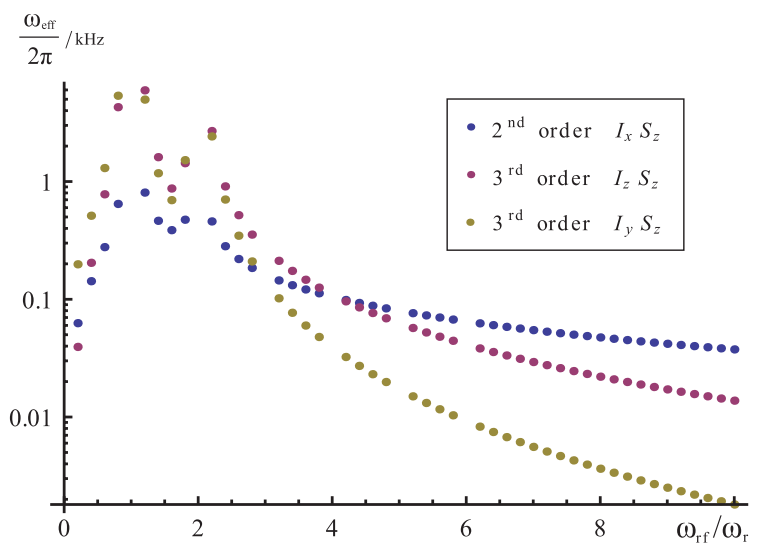

FIG. 1. Amplitudes for the second- and third-order effective Hamiltonians for a dipolarly coupled $\mathrm{CH}$ two-spin-1/2 system (see Sec. IV for details on the spin system, $\omega_{\text {aniso }}^{H} / 2 \pi=3 \mathrm{kHz}$ corresponding to $\delta_{\text {aniso }}^{C S} \sim-3.5 \mathrm{ppm}$ at a $850 \mathrm{MHz}$ spectrometer, $20 \mathrm{kHz}$ sample spinning frequency) calculated as the absolute value averaged over the powder angles $\beta_{P R}$ and $\gamma_{P R}$. Values of $\omega_{r f} / \omega_{r}$ are chosen in order to fulfill $p / q, p, q$ being integers. order terms contribute significantly to the residual Hamiltonian, however, with an effect decreasing rapidly with increasing decoupling field strength, falling to below the value of the second-order term at $\omega_{r f}=4 \omega_{r}$, i.e., $80 \mathrm{kHz} \mathrm{CW}$ decoupling for $20 \mathrm{kHz}$ spinning. The third-order terms remain in the same order of magnitude as the second-order term up till around $\omega_{r f}=10 \omega_{r}$, which underlines their importance. The $I_{y} S_{z}$ term, however, falls off more rapidly than the $I_{z} S_{z}$ term and above $\omega_{r f}=6 \omega_{r}$ it is an order of magnitude smaller than the second-order term.

We note that the analysis shown in Figure 1 does not include chemical shift offsets (i.e., $\omega_{\text {iso }}=0$ ). An offset analysis reveals that the contribution from a proton chemical shift offset to the $I_{z} S_{z}$ term is below $70 \mathrm{~Hz}$ for $\omega_{r f}>90 \mathrm{kHz}$ and $\omega_{\text {iso }} / 2 \pi<10 \mathrm{kHz}$ and even less for the $I_{y} S_{z}$ term. For a $10 \mathrm{kHz}$ offset, the contribution is in general of the same order of magnitude as the original terms (i.e., without offset), so this observation is likely to explain the weak offset dependency of the $r \mathrm{CW}$ sequences (vide infra). For the case of weak rf fields, the contribution is significantly higher, e.g., $0.6 \mathrm{kHz}$ for $\omega_{r}=1.5 \omega_{r f}$ and $\omega_{i s o} / 2 \pi=10 \mathrm{kHz}$, however, still being of similar size as the original terms.

\section{DESIGN OF HIGH-ORDER COMPENSATING DECOUPLING SEQUENCES}

In this section, we will describe how the decoupling performance may systematically be improved through use of the information about the second- and third-order residual Hamiltonians described above. These include both the pure dipoledipole coupling terms and the cross terms including effects from proton chemical shift anisotropy-being the two possibilities to reach static terms in the effective Hamiltonian which survive coherent averaging by combined MAS and CW rf irradiation.

Our aim is to refocus unwanted evolution due to the terms (see Eqs. (15) and (18))

$$
\begin{aligned}
\overline{\tilde{H}}_{x}^{(2,3)} & =\overline{\tilde{H}}^{(2)}+\overline{\tilde{H}}^{(3)} \\
& =\omega_{x}^{(2)} 2 I_{x} S_{z}+\omega_{y}^{(3)} 2 I_{y} S_{z}+\omega_{z}^{(3)} 2 I_{z} S_{z}
\end{aligned}
$$

in the effective Hamiltonian.

In the analysis, we will make use of the toggling frame approach and the semi-continuous Baker-CampbellHausdorff $(\mathrm{BCH})$ expansion. ${ }^{33}$ The toggling frame is used to account for the effect of the refocusing pulses on the effective Hamiltonians and is a rotating frame transformation of a constant angle, i.e., the flip-angle of the pulse. From the semicontinuous $\mathrm{BCH}$ evaluation, ${ }^{33}$ we make extensive use of the additivity of effective Hamiltonians

$$
\overline{\tilde{H}}^{(n)}=\frac{1}{\tau_{c}} \sum_{i=1}^{N} \tau_{i} \overline{\tilde{H}}_{i}^{(n)},
$$

where the sum is over the successive $n$th order effective Hamiltonians $\left\{\overline{\tilde{H}}_{1}^{(n)}, \ldots, \overline{\tilde{H}}_{N}^{(n)}\right\}$ of time $\tau_{i}$ and $\tau_{c}=\sum_{i=1}^{N} \tau_{i}$. We note that the simple formulation in Eq. (28) only applies when no lower-order terms are mixing into the relevant order $n$ through commutators, which in this particular case applies up to third order. In the description of the various $r \mathrm{CW}$ 
(a)

\begin{tabular}{|c|c|c|c|c|c|c|}
\hline 1 & $y$ & 2 & & & & \\
\hline$x$ & & $x$ & & & & \\
\hline \multicolumn{7}{|l|}{ (b) } \\
\hline 1 & $y$ & 2 & $x$ & 3 & $y$ & 4 \\
\hline$x$ & & $x$ & & $x$ & & $x$ \\
\hline
\end{tabular}

(c)

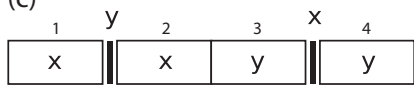

(d)

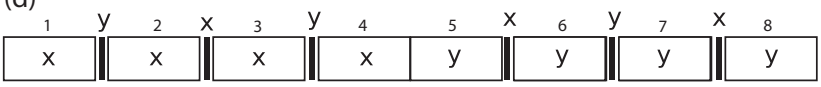

(e)

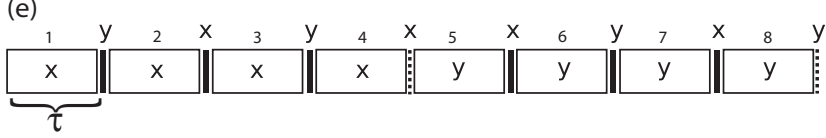

FIG. 2. Schematic representation of $r \mathrm{CW}$ decoupling sequences. Wide open boxes represent $\mathrm{CW}$ rf irradiation of phase indicated inside the square, narrow closed bars represent $180^{\circ}$ pulses, and dashed bars represent $90^{\circ}$ pulses. In the two latter cases, the phase of the pulse is given above the bar. The $180^{\circ}$ and $90^{\circ}$ pulses are applied rotor-synchronously, i.e., at $\tau=n \tau_{r}, n$ being an integer. In the text we will use the following short-hand notation for the five pulse sequences (a) $r \mathrm{CW}^{A}$, (b) $r \mathrm{CW}^{B}$, (c) $r \mathrm{CW}^{C}$, (d) $r \mathrm{CW}^{D}$, and (e) $r \mathrm{CW}^{E}$. The numbers above the $\mathrm{CW}$ decoupling elements correspond to the numbers used for the Hamiltonians in the analysis of the sequences in the text.

decoupling sequences, we will use the short-hand notation outlined in Fig. 2 using $r \mathrm{CW}^{A}$ to designate sequence $A$ in the figure and so forth.

\section{A. Design strategy 1: Refocusing}

The first element in our design strategy relies on refocusing unwanted effective Hamiltonians by inserting $\pi$ rf pulses into the $\mathrm{CW}$ irradiation in synchrony with the rotor revolution, i.e., at $\tau=n \tau_{r}$, with $n$ being an integer. We note here (as also applying to the insertion of $\pi / 2$ pulses, vide infra) that $\tau$ $\sim 100 \mu$ s appears to work well experimentally.

Using the toggling frame approach, the effective Hamiltonian for the second part of the basic $r \mathrm{CW}^{A}$ sequence (after the $\pi$ pulse; Fig. 2(a)) becomes

$$
\begin{aligned}
\overline{\tilde{H}}_{2}^{(2,3)} & =e^{-i \pi I_{y}} \overline{\tilde{H}} e^{i \pi I_{y}} \\
& =-\omega_{x}^{(2)} 2 I_{x} S_{z}+\omega_{y}^{(3)} 2 I_{y} S_{z}-\omega_{z}^{(3)} 2 I_{z} S_{z},
\end{aligned}
$$

which through the use of Eqs. (19)-(21) leads to the overall effective Hamiltonian

$$
\overline{\tilde{H}}_{1+2}^{(2,3)}=\frac{1}{2 \tau}\left(\tau \overline{\tilde{H}}_{1}+\tau \overline{\tilde{H}}_{2}\right)=\omega_{y}^{(3)} 2 I_{y} S_{z},
$$

with $\tau$ signifying the delay between the refocusing pulses.

It is seen that the $y$-phase $\pi$ pulse, as expected, refocuses the second-order $2 I_{x} S_{z}$ and third-order $2 I_{z} S_{z}$ components, leaving only the third-order $2 I_{y} S_{z}$ term in the effective Hamiltonian. This should immediately improve the decoupling significantly, not least under strong rf field conditions as terms with $\omega_{r f}^{-1}$ and $\omega_{r f}^{-2}$ dependencies are removed. It should, however, be noted that the decoupling performance still suffers from the remaining third-order term, which in many cases may be of the same order of magnitude as the canceled terms although being proportional to $\omega_{r f}^{-3}$.
To compensate for the third-order $2 I_{y} S_{z}$ term an $x$-phase $\pi$ pulse is inserted between two $r \mathrm{CW}^{A}$ sequences, leading to the $r \mathrm{CW}^{B}$ decoupling sequence (Fig. 2(b)). This sequence adds the Hamiltonian (toggling-frame, induced by the $\pi_{x}$ pulse, for the second $r \mathrm{CW}^{A}$ element)

$$
\overline{\tilde{H}}_{3+4}^{(2,3)}=-\omega_{y}^{(3)} 2 I_{y} S_{z},
$$

to the effective Hamiltonian of the full sequence, leading to

$$
\overline{\tilde{H}}_{1+2+3+4}^{(2,3)}=0 \text {. }
$$

This approach leaves the $S$ - and the $I$-spins fully decoupled to third order. For this to take effect, it requires, however, that the refocusing pulses are significantly stronger than the $\mathrm{CW}$ decoupling. This applies in particular to the $x$-phase $\pi$ pulses for which the inter-pulse spacing is quite large.

\section{B. Design strategy 2: Phase modulation}

Our second element in the design strategy involves concatenation of phase changed elements as previously employed for so-called z-rotational decoupling. ${ }^{43,44}$ Taking origin in the $r \mathrm{CW}^{A}$ sequence this materializes to the $r \mathrm{CW}^{C}$ decoupling sequence (Fig. 2(c)), where an element is inserted, in which the $\mathrm{CW}$ irradiation is of phase $y$ while the $\pi$ pulse is of phase $x$. The rationale behind this sequence may easily be seen by considering the effective Hamiltonian observed under CW $y$ phase rf irradiation,

$$
\overline{\tilde{H}}_{y}^{(2,3)}=\omega_{x}^{(2)} 2 I_{y} S_{z}-\omega_{y}^{(3)} 2 I_{x} S_{z}+\omega_{z}^{(3)} 2 I_{z} S_{z} .
$$

This expression along with the corresponding $x$-phase Hamiltonian (see Eq. (21)) leads to the following eight toggling frame Hamiltonians for the $r \mathrm{CW}^{C}$ sequence repeated two times ( $\mathrm{a}$ and $\mathrm{b}$ in the following, four Hamiltonians for each sequence with numbers corresponding to the numbers in Fig. 2(c))

$$
\begin{aligned}
& \overline{\tilde{H}}_{1 a}^{(2,3)}=\omega_{x}^{(2)} 2 I_{x} S_{z}+\omega_{y}^{(3)} 2 I_{y} S_{z}+\omega_{z}^{(3)} 2 I_{z} S_{z}, \\
& \tilde{\tilde{H}}_{2 a}^{(2,3)}=-\omega_{x}^{(2)} 2 I_{x} S_{z}+\omega_{y}^{(3)} 2 I_{y} S_{z}-\omega_{z}^{(3)} 2 I_{z} S_{z}, \\
& \overline{\tilde{H}}_{3 a}^{(2,3)}=\omega_{x}^{(2)} 2 I_{y} S_{z}+\omega_{y}^{(3)} 2 I_{x} S_{z}-\omega_{z}^{(3)} 2 I_{z} S_{z}, \\
& \overline{\tilde{H}}_{4 a}^{(2,3)}=-\omega_{x}^{(2)} 2 I_{y} S_{z}+\omega_{y}^{(3)} 2 I_{x} S_{z}+\omega_{z}^{(3)} 2 I_{z} S_{z}, \\
& \overline{\tilde{H}}_{1 b}^{(2,3)}=-\omega_{x}^{(2)} 2 I_{x} S_{z}-\omega_{y}^{(3)} 2 I_{y} S_{z}+\omega_{z}^{(3)} 2 I_{z} S_{z}, \\
& \tilde{\tilde{H}}_{2 b}^{(2,3)}=\omega_{x}^{(2)} 2 I_{x} S_{z}-\omega_{y}^{(3)} 2 I_{y} S_{z}-\omega_{z}^{(3)} 2 I_{z} S_{z}, \\
& \overline{\tilde{H}}_{3 b}^{(2,3)}=-\omega_{x}^{(2)} 2 I_{y} S_{z}-\omega_{y}^{(3)} 2 I_{x} S_{z}-\omega_{z}^{(3)} 2 I_{z} S_{z}, \\
& \overline{\tilde{H}}_{4 b}^{(2,3)}=\omega_{x}^{(2)} 2 I_{y} S_{z}-\omega_{y}^{(3)} 2 I_{x} S_{z}+\omega_{z}^{(3)} 2 I_{z} S_{z},
\end{aligned}
$$

which upon addition gives

$$
\overline{\tilde{H}}^{(2,3)}=0 .
$$

We note, however, that this averaging is obtained upon doubling the period as compared to the sequence $r \mathrm{CW}^{B}$, which in practice may lead to relatively lower decoupling capacity.

The decoupling sequence $r \mathrm{CW}^{D}$ (Fig. 2(d)) is, in analogy to the $r \mathrm{CW}^{C}$ sequence, developed by taking sequence $r \mathrm{CW}^{B}$ 
and adding the corresponding phase changed element. Up to third order, this should from an effective Hamiltonian point of view not add any extra performance relative to the basic $r \mathrm{CW}^{B}$ element under the assumption of ideal inversion pulses in which case all considered residual terms will cancel. This is confirmed by simulations, which show a slight decoupling efficiency increase under circumstances where $r \mathrm{CW}^{B}$ is less efficient, e.g., when the refocusing pulse rf field strength approaches the rf field strength of the surrounding CW irradiation (Fig. 3). This effect is not confirmed experimentally (vide infra) which may be ascribed to the very long averaging time (2 sequence elements $\sim 0.8 \mathrm{~ms}$ under $20 \mathrm{kHz}$ sample spinning conditions).

\section{Design strategy 3: $\pi / 2$ purging pulses}

Finally, we introduce $\pi / 2$ purging pulses as an element to improve the decoupling performance. In multiple-spin systems, terms of the type $4 I_{x} J_{x} S_{z}$ may appear as, e.g., revealed by an analysis of the effective Hamiltonian for a $\mathrm{CH}_{2}$ spin system. Such an analysis introduces third-order cross terms between $\tilde{H}_{I S}^{D}, \tilde{H}_{I J}^{D}$, and $\tilde{H}_{J}^{C S A}$ (likewise cross terms between $\tilde{H}_{J S}^{D}, \tilde{H}_{I J}^{D}$, and $\tilde{H}_{I}^{C S A}$ ) proportional to $4 I_{x} J_{x} S_{z}, 4 I_{y} J_{y} S_{z}$, and $4 I_{z} J_{z} S_{z}$. Inversion pulses applied on the proton rf channel (proton spins denoted $I$ and $J$ ) will leave such terms unperturbed, while incorporation of $\pi / 2$ pulses may be used to purge or scale such terms.

Under the assumption that all terms in the third-order effective Hamiltonian except those mentioned above are refocused by the $r \mathrm{CW}^{B}$ sequence (or similarly block 1-4 of sequence $r \mathrm{CW}^{D}$ ) and that the same holds true for the phase-shifted sequence (second half of sequence $r \mathrm{CW}^{D}$, block 5-8), the effect of incorporating $\pi / 2$ purging pulses in sequence $r \mathrm{CW}^{D}$ (resulting in sequence $r \mathrm{CW}^{E}$ ) can be evaluated considering the Hamiltonians

$$
\begin{aligned}
& \overline{\tilde{H}}_{x}=\omega_{x} 4 I_{x} J_{x} S_{z}+\omega_{y} 4 I_{y} J_{y} S_{z}+\omega_{z} 4 I_{z} J_{z} S_{z}, \\
& \tilde{\tilde{H}}_{y}=\omega_{y} 4 I_{x} J_{x} S_{z}+\omega_{x} 4 I_{y} J_{y} S_{z}+\omega_{z} 4 I_{z} J_{z} S_{z},
\end{aligned}
$$

with $\omega_{x}, \omega_{y}$, and $\omega_{z}$ being the relevant amplitude factors. These Hamiltonians describe the effective Hamiltonians originating from the aforementioned terms, which are unaffected by the inversion pulses.

A toggling frame approach is used for three consecutive repetitions of sequence $r \mathrm{CW}^{E}$ (denoted $a, b$, and $c$ in the following) considering only the non-refocused terms of the Hamiltonian (i.e., the terms in Eqs. (27) and (28)) and the $\pi / 2$ purging pulses. That is, each element $(a, b$, and $c)$ consists of $\overline{\tilde{H}}_{x}$, a $(\pi / 2)_{x}$ pulse, $\overline{\tilde{H}}_{y}$, and a $(\pi / 2)_{y}$ pulse. The following toggling frame Hamiltonians are obtained

$$
\begin{aligned}
& \overline{\tilde{H}}_{x, a}=\omega_{x} 4 I_{x} J_{x} S_{z}+\omega_{y} 4 I_{y} J_{y} S_{z}+\omega_{z} 4 I_{z} J_{z} S_{z}, \\
& \overline{\tilde{H}}_{y, a}=\omega_{y} 4 I_{x} J_{x} S_{z}+\omega_{x} 4 I_{z} J_{z} S_{z}+\omega_{z} 4 I_{y} J_{y} S_{z}, \\
& \overline{\tilde{H}}_{x, b}=\omega_{x} 4 I_{z} J_{z} S_{z}+\omega_{y} 4 I_{x} J_{x} S_{z}+\omega_{z} 4 I_{y} J_{y} S_{z}, \\
& \overline{\tilde{H}}_{y, b}=\omega_{y} 4 I_{y} J_{y} S_{z}+\omega_{x} 4 I_{x} J_{x} S_{z}+\omega_{z} 4 I_{z} J_{z} S_{z}, \\
& \overline{\tilde{H}}_{x, c}=\omega_{x} 4 I_{y} J_{y} S_{z}+\omega_{y} 4 I_{z} J_{z} S_{z}+\omega_{z} 4 I_{x} J_{x} S_{z}, \\
& \overline{\tilde{H}}_{y, c}=\omega_{y} 4 I_{z} J_{z} S_{z}+\omega_{x} 4 I_{y} J_{y} S_{z}+\omega_{z} 4 I_{x} J_{x} S_{z} .
\end{aligned}
$$

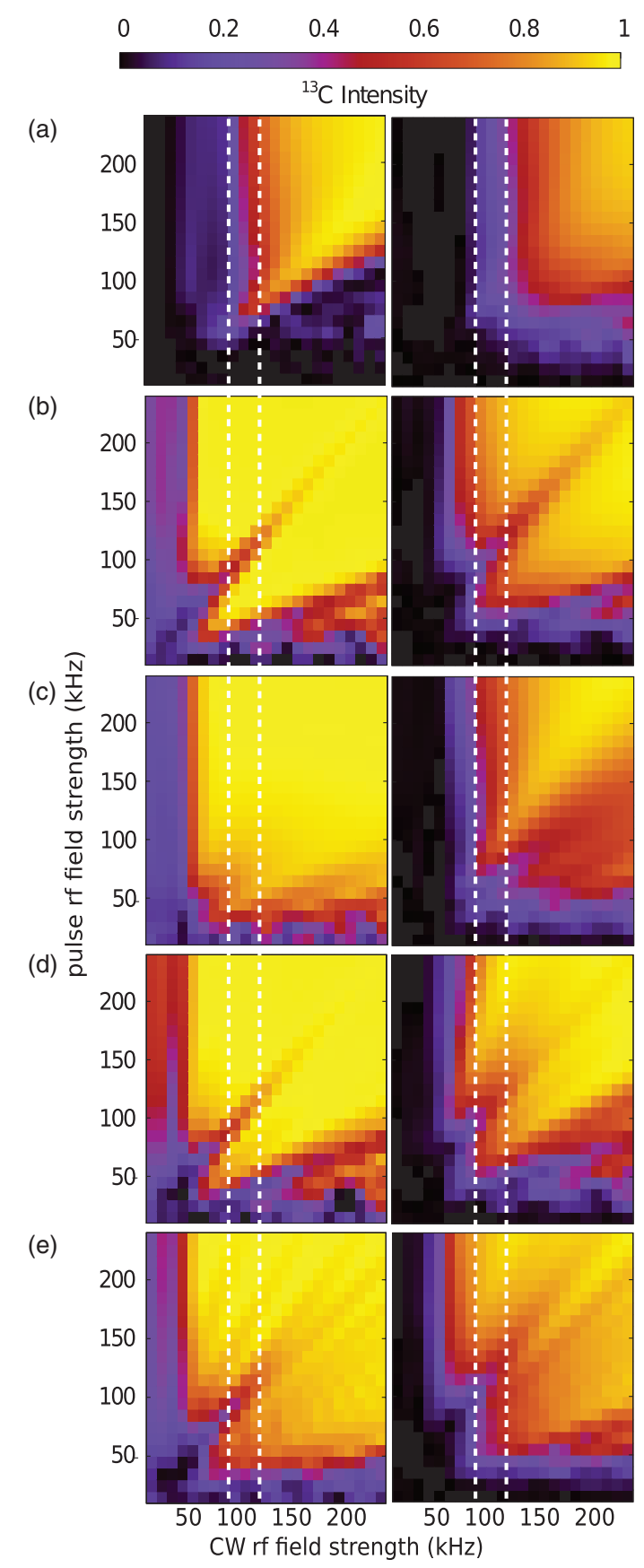

FIG. 3. Numerically calculated intensities observed after $10 \mathrm{~ms}$ free precession in the ${ }^{13} \mathrm{C}$ free-induction decays obtained for a powder of a $\mathrm{CH}$ (left column) and a $\mathrm{CH}_{2}$ (right column) spin systems (on-resonance on the ${ }^{13} \mathrm{C}$ and ${ }^{1} \mathrm{H}$ (average) spins) using (a) $r \mathrm{CW}^{A}$, (b) $r \mathrm{CW}^{B}$, (c) $r \mathrm{CW}^{C}$, (d) $r \mathrm{CW}^{D}$, and (e) $r \mathrm{CW}^{E}$ decoupling sequences with rf field strengths on the refocusing/purging pulses and $\mathrm{CW}$ irradiation. The dashed white lines indicate $\mathrm{CW}$ rf field strengths of 90 and $120 \mathrm{kHz}$. The spinning frequency is $20 \mathrm{kHz}$ while the spectrometer frequency is $850 \mathrm{MHz}$. Parameters otherwise as described in Sec. IV.

The effective Hamiltonian over all six elements (i.e., three repetitions of $r \mathrm{CW}^{E}$ ) becomes

$$
\overline{\tilde{H}}=\frac{1}{3}\left(\omega_{x}+\omega_{y}+\omega_{z}\right)\left(4 I_{x} J_{x} S_{z}+4 I_{y} J_{y} S_{z}+4 I_{z} J_{z} S_{z}\right)
$$


That is, regarding the protons, an isotropic Hamiltonian is obtained. This will, depending on $\omega_{x}, \omega_{y}, \omega_{z}$, downscale the Hamiltonian.

\section{MATERIALS AND METHODS}

All numerical simulations were carried out using the SIMPSON simulation software package, ${ }^{45-47}$ supplemented with the use of the program SIMMOL $^{48}$ for implementation and orientation tests of anisotropic interaction tensors, and REPULSION for powder averaging (66 $\alpha_{C R}, \beta_{C R}$ angles). ${ }^{49}$ The two spin systems analyzed were a typical aliphatic ${ }^{13} \mathrm{C}_{\alpha}$ $\mathrm{CH}$ group and a typical aliphatic $\mathrm{CH}_{2}$-group with the tetrahedral angle between the $\mathrm{CH}$ vectors. The following parameters were used: $\delta_{H}^{C S A}=-2975 \mathrm{~Hz}$ (corresponding to -3.5 ppm at $850 \mathrm{MHz}), \delta_{C}^{C S A}=0 \mathrm{~Hz}, \eta^{C S A}=0, \delta_{H 1}^{i s o}=0 \mathrm{~Hz}, \delta_{H 2}^{i s o}$ $=200 \mathrm{~Hz}, \quad \delta_{C}^{i s o}=0 \mathrm{~Hz}, \omega_{H C}^{D} / 2 \pi=23.3 \mathrm{kHz}, \omega_{H H}^{D} / 2 \pi$ $=21.3 \mathrm{kHz}, \omega_{H C}^{J} / 2 \pi=130 \mathrm{~Hz}$, and $\omega_{H H}^{J} / 2 \pi=13 \mathrm{~Hz}$. All calculations assumed $20 \mathrm{kHz}$ sample spinning and a spectrometer frequency of $850 \mathrm{MHz}$. Analytical calculations of effective Hamiltonians were in part assisted by calculations using Mathematica. ${ }^{50}$

Experiments were conducted on a Bruker Avance II $700 \mathrm{MHz}$ wide-bore spectrometer (Bruker Biospin, Rheinstetten, Germany) equipped with a $2.5 \mathrm{~mm}$ triple-resonance probe (data in Fig. 5) and on a Varian NMR System 850 $\mathrm{MHz}$ spectrometer (Agilent Technologies, Santa Clara, California) equipped with a $1.2 \mathrm{~mm}$ UltraFastMAS T3 probe in double resonant (HC) mode (data in Figs. 6 and 7). On the Bruker instrument, decoupling sequences were implemented using cpd programming in topspin and data obtained for uniformly ${ }^{13} \mathrm{C},{ }^{15} \mathrm{~N}$-labeled glycine (99\% labeling) (Isotec, Sigma-Aldrich). On the Varian instrument, decoupling experiments were implemented using asynchronous pulse programming and data were obtained for glycine ${ }^{13} \mathrm{C}$-labeled on $\mathrm{C}_{\alpha}$ (25\% labeling) (Sigma-Aldrich, prepared from $99 \% \mathrm{C}_{\alpha}$ enriched glycine by co-crystallization with natural abundance glycine to achieve an overall enrichment of 25\%). For all $r \mathrm{CW}$ sequences $\tau=2 \tau_{r}$ was around $100 \mu \mathrm{s}$, giving rise to cycle times ranging from $200 \mu \mathrm{s}$ for $r \mathrm{CW}^{A}$ up to $800 \mu \mathrm{s}$ for $\mathrm{rCW}^{D}$ and $\mathrm{rCW}^{E}$. In all cases excitation was performed using standard cross polarization (CP) MAS protocols, and the data were processed using topspin (Bruker Biospin, Rheinstetten, Germany) and the matNMR toolbox ${ }^{51}$ for MATLAB.

\section{CONSIDERATIONS REGARDING IMPLEMENTATION OF rCW DECOUPLING}

Prior to implementation of the $r \mathrm{CW}$ sequences a number of parameters of relevance for the performance should be addressed. In the following we assume that the synchronization conditions addressed in the effective Hamiltonian analysis are fulfilled. From the derived effective Hamiltonians, we know that non-refocused second-order terms enter with the coefficient

$$
\frac{\omega_{r f}}{n^{2} \omega_{r}^{2}-\omega_{r f}^{2}}
$$

while the third-order terms enter with the coefficients

$$
\frac{n m \omega_{r}^{2}+3 \omega_{r f}^{2}}{n^{2} m^{2} \omega_{r}^{4}-\left(n^{2}+m^{2}\right) \omega_{r}^{2} \omega_{r f}^{2}+\omega_{r f}^{4}}
$$

and

$$
\frac{\omega_{r} \omega_{r f}}{n^{2} m^{2} \omega_{r}^{4}-\left(n^{2}+m^{2}\right) \omega_{r}^{2} \omega_{r f}^{2}+\omega_{r f}^{4}} .
$$

It is anticipated that the refocusing pulses significantly reduce these scaling factors as described above. In this regard, it seems relevant to consider the size of the residual terms under different conditions. As long as $\omega_{r f} \gg \omega_{r}$, the rf irradiation dominates and the dependencies become proportional to $1 / \omega_{r f}, 1 / \omega_{r f}^{2}$, and $1 / \omega_{r f}^{3}$, respectively, for the three terms addressed above. This reveals, as expected, that the decoupling becomes more effective with increasing power for the $\mathrm{CW}$ rf irradiation. If, on the other hand, $\omega_{r}$ approaches $\omega_{r f}$ through increased spinning speed or decreased decoupling power, terms with significantly smaller denominators occur and insertion of refocusing and purging pulses becomes increasingly important. Figure 1 addresses this more complicated case as well as the regime, where $\omega_{r f}$ dominates the denominator.

From this analysis, it is clear that $\mathrm{CW}$ decoupling is most effective for $\omega_{r f}>3 \omega_{r}$, since the third-order term otherwise will dominate. This condition gives an upper limit to the spinning speed for limited rf power, that is, if $120 \mathrm{kHz}$ as an example is the upper limit of the rf power available, $r \mathrm{CW}$ decoupling is a priori only relevant for spinning speeds up to $40 \mathrm{kHz}$. However, it is desirable to apply the refocusing pulses with higher power than applied for the $\mathrm{CW}$ irradiation in order to refocus residual terms in the effective Hamiltonian most effectively, still keeping a low average rf decoupling power level. Therefore even with $200 \mathrm{kHz}$ rf field strength, spinning speeds below $30 \mathrm{kHz}$ are of greatest interest. For $20 \mathrm{kHz}$ spinning, the most promising range of $\mathrm{CW}$ rf amplitudes will be in the order of $60-100 \mathrm{kHz}$, avoiding the recoupling conditions at 60,80 , and $100 \mathrm{kHz}$.

To address in more detail the applicable range of rf field strengths, Fig. 3 analyzes through SIMPSON ${ }^{45}$-based numerical simulations the interplay between the refocusing/purging rf pulse strength and the rf field strength of the $\mathrm{CW}$ decoupling for the five $r \mathrm{CW}$ sequences for $\mathrm{CH}$ - and $\mathrm{CH}_{2}$-spin systems at $20 \mathrm{kHz}$ MAS. First, the plots show the increased efficiency of refocusing all the terms of the effective Hamiltonian, when comparing sequence $r \mathrm{CW}^{A}$ with the other $r \mathrm{CW}$ sequences, i.e., refocusing of the $I_{y} S_{z}$ term significantly increases the decoupling performance at $\mathrm{CW}$ rf field strengths of 50-150 kHz. Second, for all sequences which include refocusing pulses of phase similar to the phase of the $\mathrm{CW}$ irradiation, a clear minimum is found for matching rf field strengths (i.e., pulse and $\mathrm{CW}$ ) as seen for sequence $r \mathrm{CW}^{B}, r \mathrm{CW}^{D}$, and $r \mathrm{CW}^{E}$. This leaves us with two regimes: $\omega_{r f}^{\text {pulse }}>\omega_{r f}^{C W}$ and $\omega_{r f}^{\text {pulse }}<\omega_{r f}^{C W}$. Of these, the first provides the most attractive condition since it leads to a lower average rf field strength (in the typical case where the pulses are by far shorter than the $\mathrm{CW}$ periods). 


\section{COMPARATIVE ANALYSIS OF DECOUPLING SEQUENCES}

In this section, we present a comparative analysis of $r \mathrm{CW}$ and state-of-the-art decoupling sequences in the high-power rf field regime discussed above. We should in this context note that the effective Hamiltonian analysis in Fig. 1 in addition to this regime also reveals a regime with potential for low-power decoupling at high spinning frequencies as earlier elaborated on by Ishii and co-workers ${ }^{52}$ and Ernst et al..$^{35}$ This regime will not be analyzed in further detail in the present work.

\section{A. Numerical evaluation of decoupling performance for $\mathrm{CH}$ and $\mathrm{CH}_{2}$ spin systems}

Aimed at documenting the applicability of our strategy for systematic design of decoupling sequences through refocusing of high-order dipolar-coupling- and chemicalshielding-anisotropy-induced static terms in the effective Hamiltonian of $\mathrm{CW}$ decoupling experiments and compare the performance of the resulting $r \mathrm{CW}$ sequences with state-ofthe-art decoupling experiments, we performed a series of simulations using the SIMPSON program. ${ }^{45}$ To cover the cases addressed analytically and provide a good basis for biological solid-state NMR applications, we performed simulations for spin systems resembling $\mathrm{C}_{\alpha} \mathrm{H}$ and $\mathrm{C}_{\alpha} \mathrm{H}_{2}$ spin systems as described in Sec. IV. On this basis, Fig. 4 compares the free-induction-decays (FIDs) observed under decoupling conditions for our five different $r \mathrm{CW}$ sequences as compared with CW and optimized TPPM, ${ }^{7}$ SPINAL- $64,{ }^{8}$ tangentially swept TPPM (SW ${ }_{f}^{\text {tan }}$-TPPM) ${ }^{12}$ and linearly swept SPINAL $\left(\mathrm{SW}_{f}^{\text {lin }}(32)\right.$-SPINAL-32) ${ }^{14}$ sequences for $\mathrm{CH}$ - and $\mathrm{CH}_{2}$-spin systems with and without taking into account the ${ }^{1} \mathrm{H}-{ }^{1} \mathrm{H}$ homonuclear dipolar coupling in the latter case.

From Fig. 4, it becomes evident that all $r \mathrm{CW}$ sequences, except the basic element $r \mathrm{CW}^{A}$, show near-to-ideal decoupling for the $\mathrm{CH}$-spin system, as does it for the $\mathrm{CH}_{2}$-spin system without ${ }^{1} \mathrm{H}-{ }^{1} \mathrm{H}$ couplings included in the calculation. It is seen in both cases that all $r \mathrm{CW}$ sequences with the exception of $r \mathrm{CW}^{A}$ performs better than the techniques included in the comparison through their current ranking as the most powerful decoupling methods. We note that only minor differences are seen between the original TPPM and SPINAL sequences and their swept variants in this case, where resonance offsets are not considered distinguishing parameters. For all sequences, a considerable drop in performance is seen for the $\mathrm{CH}_{2}$-spin system in the ordinary case with the homonuclear coupling included in the calculations (Fig. 4, bottom row). Looking at the position of the red spot, marking the onresonance ${ }^{13} \mathrm{C}$ signal intensity at $10 \mathrm{~ms}$, it is clear, however, that sequences such as $r \mathrm{CW}^{B}, r \mathrm{CW}^{D}$, and $r \mathrm{CW}^{E}$ demonstrate very good decoupling along with the SW-SPINAL sequence as the best among the analyzed previous methods. In this context, we remind that the performance of the $r \mathrm{CW}$ decoupling sequences depends on the ideality of the refocusing pulses, which for the $\mathrm{CH}$ spin system leads to perfect decoupling if infinitely strong and short refocusing pulses are used.

On the basis of the simulations in Fig. 4, the three strategies outlined in Sec. III to improve decoupling performance can be evaluated. The refocusing strategy seems to perform excellently in all cases with marked improvements on going from $\mathrm{CW}$ decoupling to decoupling using sequence $r \mathrm{CW}^{A}$ and further with remarkable improvements using sequence $r \mathrm{CW}^{B}$. This might not appear surprising, since high-power

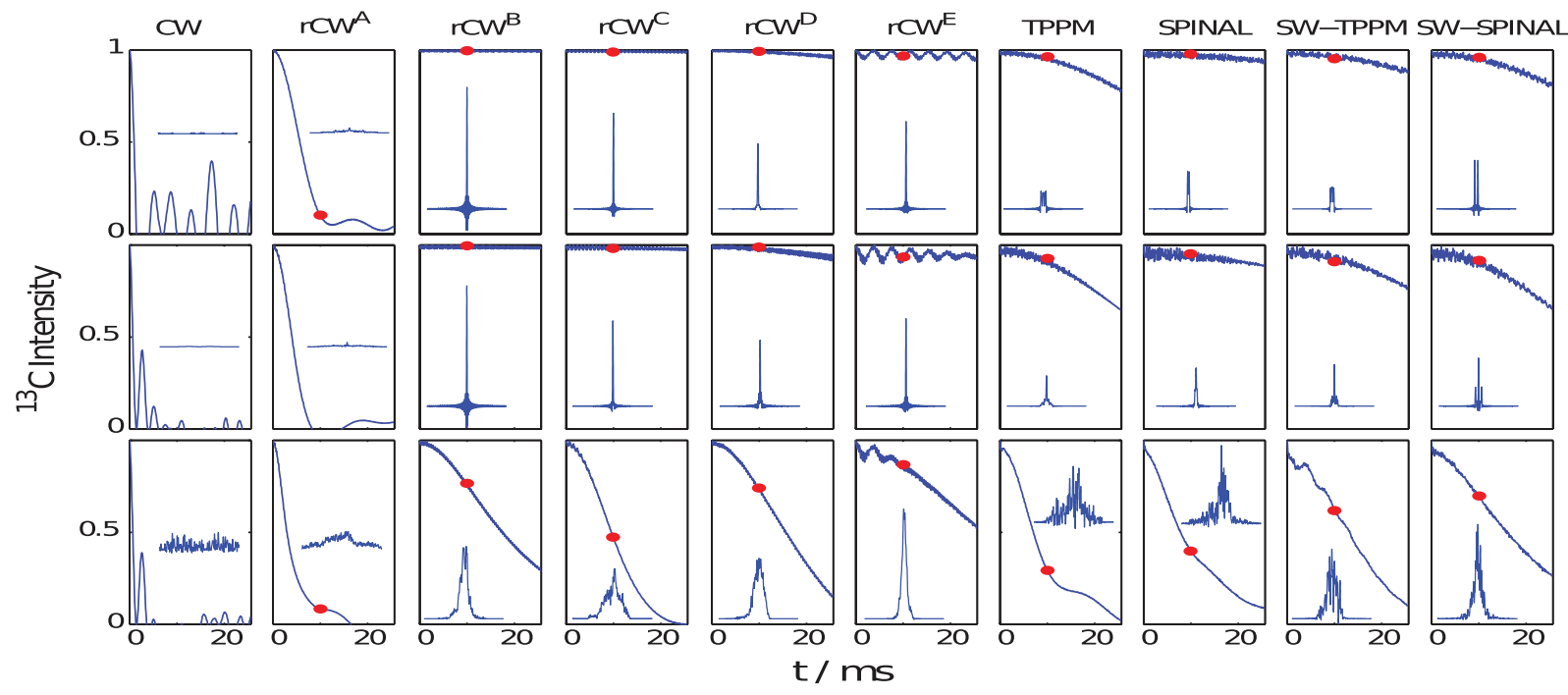

FIG. 4. Numerically calculated ${ }^{13} \mathrm{C}$ free-induction decays (on-resonance on the ${ }^{13} \mathrm{C}$ and ${ }^{1} \mathrm{H}$ (average frequency) spins) comparing the performance of different decoupling sequences (see labels in top of the figure; the $r \mathrm{CW}$ sequences marked with superscripts A-E referring to Fig. 2) using an average rf field strength of $93.7 \mathrm{kHz}$ (for $r \mathrm{CW}$ corresponding to $90 \mathrm{kHz}$ for the $\mathrm{CW}$ blocks and $222 \mathrm{kHz}$ for the refocusing pulses). For TPPM and SPINAL (and their swept variants), the pulse length and phase shift have been optimized for the individual cases. The red dots indicate the intensity observed at $10 \mathrm{~ms}$ to provide an easy measure on the decoupling performance. The comparison addresses spin systems with and without proton-proton dipolar couplings: $\mathrm{CH}_{\text {-spin }}$ system (top), $\mathrm{CH}_{2}$-spin system without including the ${ }^{1} \mathrm{H}-{ }^{1} \mathrm{H}$ dipolar coupling (middle), and a normal $\mathrm{CH}_{2}$-spin system including the homonuclear coupling (bottom). Spinning frequency of $20 \mathrm{kHz}$, spectrometer frequency of $850 \mathrm{MHz}$. The small insets show the corresponding spectra (a spectral width of $\pm 100 \mathrm{~Hz}$ is shown, total acquisition time is $496 \mathrm{~ms}$, spectra in bottom row has been scaled up by a factor of ten). 
"close-to-ideal" refocusing pulses (222 kHz rf field strength) have been applied without considering instrumental errors, phase transients, rf inhomogeneity, etc. Proceeding to concatenation of phase-changed elements, it is seen that this strategy is indeed successful in removing all considered residual second- and third-order Hamiltonians for the $\mathrm{CH}$ spin system as revealed by comparing the performance of sequences $r \mathrm{CW}^{A}$ and $r \mathrm{CW}^{C}$. However, in particular for the $\mathrm{CH}_{2}$ spin system including homonuclear dipolar couplings, the $r \mathrm{CW}^{C}$ sequence appears less efficient than the $r \mathrm{CW}^{B}$ sequence. Addressing the phase-change strategy for the more advanced sequence $r \mathrm{CW}^{B}$ (leading to the decoupling sequence $r \mathrm{CW}^{D}$ ), it is seen that this does not provide significant advantages, which presumably is a consequence of the intrinsic high efficiency of sequence $r \mathrm{CW}^{B}$ and the long averaging time of sequence $r \mathrm{CW}^{D}$ (vide supra).

The $\pi / 2$-purging pulses do, on the expense of unintended low-frequency oscillations, increase the decoupling efficiency as evidenced by comparing the performance of the sequences $r \mathrm{CW}^{B}$ and $r \mathrm{CW}^{E}$. This applies in particular for the $\mathrm{CH}_{2}$ system including homonuclear dipolar coupling between the protons, being fully consistent with our analysis in Sec. III. The high-frequency oscillations induced by the $\pi / 2$-purging pulses are of frequencies $156.25 \mathrm{~Hz}$ and $1.25 \mathrm{kHz}$ (not visible in the figure). The fast oscillation frequency matches the refocusing over each $\pi / 2$-purging pulse and the slow oscillation corresponds to refocusing over $3.2 \mathrm{~ms}$, i.e., the 4 sequence elements. According to our simulations, these oscillations do not pose a problem after Fourier transformation; they give rise to vanishingly small peaks (intensity $5 \times 10^{-5}$ of the main signal) at the given frequencies and judge from the spectral peak insert, the oscillations does not affect the line shape.

Under the finite, but strong ( $222 \mathrm{kHz}$ rf field strength), $\pi$ and $\pi / 2 \mathrm{rf}$ pulse conditions, the simulations for all $r \mathrm{CW}$ sequences except $r \mathrm{CW}^{A}$ show improved decoupling compared to state-of-the-art efficient decoupling sequences as here represented by the $\mathrm{SW}_{f}$-TPPM and $\mathrm{SW}_{f}$-SPINAL. However, with the exception of the $r \mathrm{CW}^{C}$ sequence, the $r \mathrm{CW}$ sequences rely on close-to-ideal performance of the refocusing pulses in the regime of modest (i.e., $90-120 \mathrm{kHz}$ ) rf fields for the $\mathrm{CW}$ irradiation. According to simulations of the $10 \mathrm{~ms}$ FID intensity for the $r \mathrm{CW}$ sequences for varying refocusing/purging pulse and CW rf field strength (Fig. 3), the difference between the $r \mathrm{CW}^{C}$ sequence and the other $r \mathrm{CW}$ sequences is the performance minimum for matching $\mathrm{CW}$ and refocusing pulse rf field strength for all but the $r \mathrm{CW}^{A}$ and $r \mathrm{CW}^{C}$ sequences. (Among these mainly the $r \mathrm{CW}^{C}$ sequence is of interest due to lower demands to overall rf field strengths.) This minimum is not surprising, since the sequences approach the $r \mathrm{CW}^{A}$ sequence under this condition. From the simulations, it appears that all sequences converge towards a stable high decoupling performance for refocusing pulse rf field strengths above 150 $\mathrm{kHz}$ for modest $\mathrm{CW}$ rf field strengths in the order of 90$120 \mathrm{kHz}$. As such, the requirement for high-power refocusing pulses does not seem pronounced. However, this applies only when instrumental errors such as phase transients and rf inhomogeneity are not included in the simulations.

Experimentally $r \mathrm{CW}$ decoupling may pose the need for quite high power for the refocusing/purging rf pulses
( $\gtrsim 200 \mathrm{kHz}$ ). This may obviously be a limiting factor for probes with insufficient rf performance (typically for large rotor diameters), but feasible for state-of-the-art fast-spinning probes considering the low duty cycle of these intense $\mathrm{rf}$ pulses. For lower-power applications we recommend the $r \mathrm{CW}^{C}$ (see Sec. VI B) that does not suffer from reduced performance in the regime where the rf field strengths for pulses and $\mathrm{CW}$ irradiations approaches each other.

\section{B. Experimental analysis of tolerances towards resonance offsets, chemical shift anisotropy, and if power variation for decoupling using modest rf fields}

To explore the broadbandedness and tolerance towards rf field mismatch/variation, Figure 5 compares the performance of various decoupling sequences experimentally using $140 \mathrm{kHz}$ rf field strength and $20 \mathrm{kHz}$ spinning for observation of the ${ }^{13} \mathrm{C}_{\alpha}$ resonance in uniformly ${ }^{13} \mathrm{C}$-labeled sample of glycine on a $700 \mathrm{MHz}$ spectrometer (cf. Sec. IV). In this analysis, we take advantage of the modest requirements of the $r \mathrm{CW}^{C}$ with respect to the power of the refocusing pulses. For comparison, optimized TPPM, ${ }^{7}$ SPINAL64, ${ }^{8}$ $\mathrm{SW}_{f}^{\text {tan }}$-TPPM, ${ }^{12}$ and $\mathrm{SW}_{f}^{\text {lin }}(32)$-SPINAL-32 ${ }^{14}$ were included in the analysis. We note that the $\mathrm{XiX}^{9}$ decoupling sequence was not included, since preliminary tests showed that it did not perform better than the other sequences, as also reported in Ref. 16. The experimental analysis demonstrates that the $r \mathrm{CW}^{C}$ decoupling sequence is very robust and well performing. The sequence outperforms the commonly used

(a)

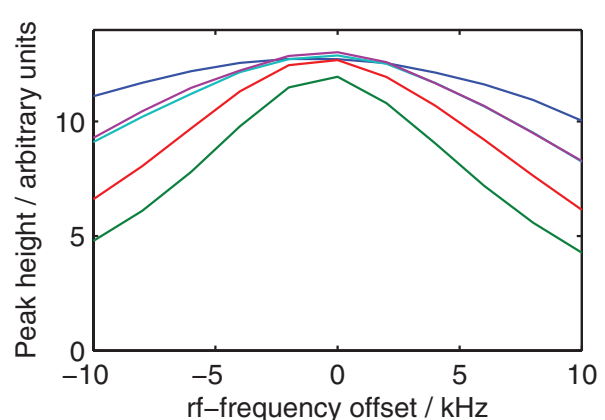

(b)

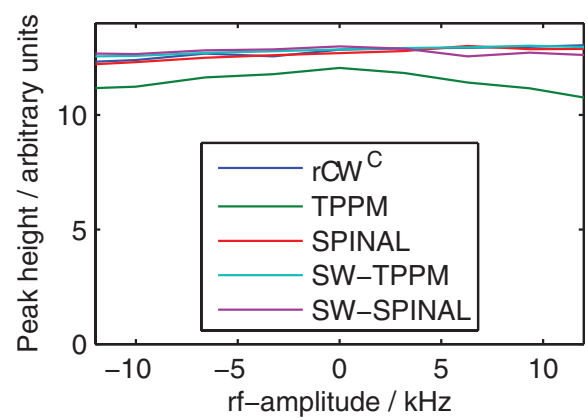

FIG. 5. Experimental analysis of $r \mathrm{CW}^{C}$ and state-of-the-art decoupling sequences addressing (a) broadbandedness with respect to $\mathrm{rf}$ offset and (b) variation of the rf amplitude as observed (peak height) for the ${ }^{13} \mathrm{C}_{\alpha}$-spin of $U-{ }^{13} \mathrm{C},{ }^{15} \mathrm{~N}$-labeled glycine under conditions of $20 \mathrm{kHz}$ sample spinning ( $2.5 \mathrm{~mm}$ rotor) on a Bruker Avance II $700 \mathrm{MHz}$ NMR spectrometer. For direct comparison all decoupling sequences used $140 \mathrm{kHz}$ rf field strength (for $r \mathrm{CW}^{C}$ both for the $\mathrm{CW}$ and refocusing pulses; for the other sequences the pulse length and phase shift have been individually optimized). 
TPPM and SPINAL64 sequences with respect to both offset and rf variation robustness, however, with the observation that for on-resonance irradiation conditions the performance is marginally lower than the swept versions of TPPM and SPINAL. In this comparison, however, it is important to stress that the $r \mathrm{CW}$ sequences are easier (i.e., faster) to set up experimentally than all the other decoupling sequences. This applies since all parameters can be independently optimized and in essence the only parameter, which really needs optimization is the $\mathrm{CW}$ rf field strength assuming that the widths of the refocusing/purging pulses are known from trivial pulse calibrations (vide infra).

\section{Performance of $r \mathrm{CW}$ decoupling with strong refocusing pulses}

To extend our performance analysis to decoupling sequences in the regime of high-power refocusing pulses and investigate the efforts needed for experimental optimization, we have undertaken both numerical and experimental comparisons of $r \mathrm{CW}$ decoupling with various state-of-the-art decoupling methods under conditions of $20 \mathrm{kHz}$ spinning and $222 \mathrm{kHz}$ rf field strength for the refocusing pulses. The SIMPSON simulations, in this case, addressed a spin system resembling a $\mathrm{C}_{\alpha} \mathrm{H}_{2}$-group of glycine (see $\mathrm{Sec}$. IV) under consideration of 5\% rf field inhomogeneity, being matched by experiments on $25 \%{ }^{13} \mathrm{C}_{\alpha}$-labeled glycine obtained using a $850 \mathrm{MHz}$ spectrometer equipped with a $1.3 \mathrm{~mm}$ probe (see Sec. IV for details).

\section{Optimization of the decoupling sequences}

An important issue in practical applications is the ease by which the decoupling sequences are optimized experimentally as their performance is critically dependent on this. This implies that small deviations from the optimal parameters may significantly reduce the performance relative to the optimized behavior as described here and elsewhere for previous sequences. The presented $r \mathrm{CW}$ decoupling experiments are easy to set up. This involves the choice of sequence in terms of refocusing/purging pulses, decision on the refocusing/purging pulse separation $\tau$ (where an integral number of rotor periods being close to $100 \mu$ s appears to be optimal), and calibration of the pulse width for the refocusing/purging pulses. With this the optimization only involves the rf field strength of the $\mathrm{CW}$ rf irradiation as illustrated numerically and experimentally in Figs. 6(a) and 6(b), respectively, for the $r \mathrm{CW}$ pulse sequences in Fig. 2. In both cases we used $222 \mathrm{kHz}$ rf field strength for

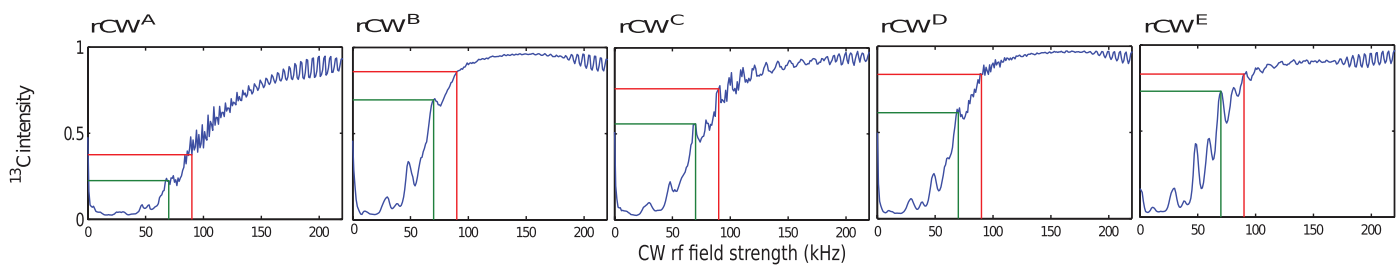

(a)
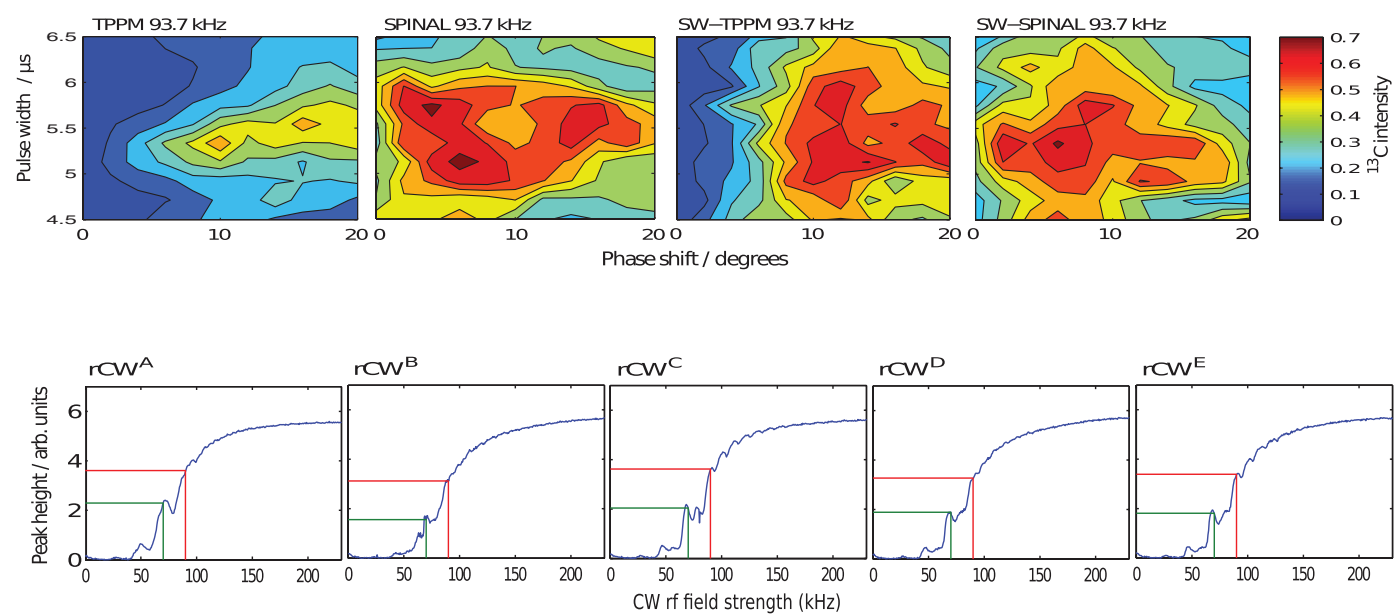

(b)
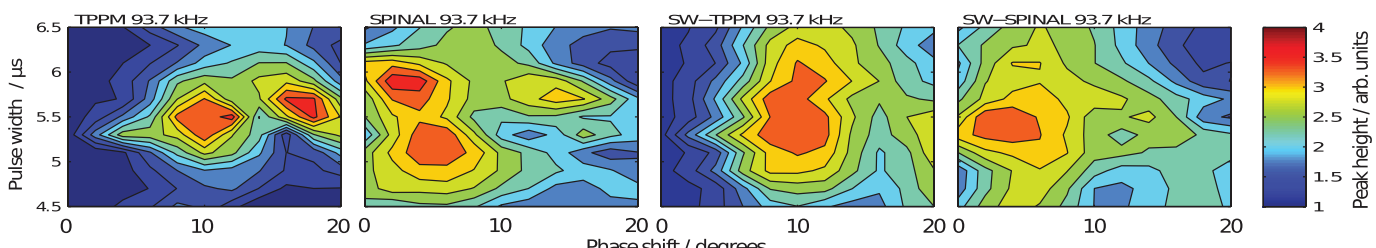

FIG. 6. Numerical (a) and experimental (b) optimization of the decoupling performance of various $r \mathrm{CW}$ decoupling sequences (222 kHz rf field strength for the refocusing/purging pulses), TPPM, SPINAL, $\mathrm{SW}_{f}$-TPPM, and $\mathrm{SW}_{f}$-SPINAL obtained for a typical $\mathrm{CH}_{2}$-spin system under conditions of an $850 \mathrm{MHz} \mathrm{NMR}$ instrument and $20 \mathrm{kHz}$ sample spinning (the experiments were obtained for $25 \%{ }^{13} \mathrm{C}_{\alpha}$-enriched glycine). In both cases the top row illustrates the normalized intensity (a) or the peak height (b) obtained using the $r \mathrm{CW}$ sequences in Fig. 2, while the lower row shows the optimization of the pulse and phase shift angle for TPPM, SPINAL, $\mathrm{SW}_{f}$-TPPM, and $\mathrm{SW}_{f}$-SPINAL sequences using an rf field strength of $93.7 \mathrm{kHz}$, corresponding to the red lines in the top row. The green lines indicate the $70 \mathrm{kHz}$ local optimum. 
the refocusing/purging pulses, corresponding to pulse widths of $t_{\pi}=2.25 \mu \mathrm{s}$.

It is evident that all but the $r \mathrm{CW}^{A}$ pulse sequence provides fairly good and converging decoupling performance using rf field amplitudes in the order of $120-140 \mathrm{kHz}$ or lower, with the experiments calling for slightly higher amplitudes than the simulations. Except for the local optimum at $\sim 70$ $\mathrm{kHz}$, the simulations and especially the experiments show reasonably steady increases in decoupling efficiency with the $\mathrm{CW}$ rf-field strength and a narrow optimization around the desired decoupling power level will be sufficient to optimize the $r \mathrm{CW}$ decoupling. We note that the optimization curves for the $r \mathrm{CW}$ sequences agree well with the expectations from the theoretical analysis in Fig. 1. The local optimum around $35 \mathrm{kHz}$ CW decoupling agrees with the dip in the size of $\omega_{\text {eff }}$ at $\omega_{r f} / \omega_{r}$ $\sim$ 1.75. The general increase in decoupling efficiency from 50 $\mathrm{kHz}$ and up agrees with the general decrease of $\omega_{\text {eff }}$ for $\omega_{r f} / \omega_{r}$ $>2.5$. Finally, the local decoupling efficiency minima match the recoupling conditions $\omega_{\text {eff }}$ for $\omega_{r f} / \omega_{r} \in$ integers.

In contrast to the simple and predictable optimal conditions for the $r \mathrm{CW}$ decoupling, it appears fairly complicated to optimize the conventional decoupling experiments as illustrated by pulse width vs pulse phase shift contour plots in Fig. 6 obtained by numerical simulations and experiments for TPPM, SPINAL, $\mathrm{SW}_{f}$-TPPM, and $\mathrm{SW}_{f}$-SPINAL. In all cases, we observe complicated optimization patterns as previously demonstrated for TPPM in Refs. 53 and 56. In the numerical simulations, it appears that both of the swept sequences reduce the sharpness of the optimal conditions as compared to the original sequences (TPPM and SPINAL). The experimental data confirm the broadening of the optimum conditions for the swept sequences, but the decoupling efficiency is not increased. The broadening may, however, in the cases where thorough optimizations are not an option lead to increased decoupling efficiency.

\section{Decoupling sequences using 93.7 and $70 \mathrm{kHz}$ average rf field strength}

To explore in more detail the experimental performance of the optimized decoupling sequences corresponding to the case of $93.7 \mathrm{kHz}$ average rf field strength, Fig. 7(a) shows line shapes as obtained experimentally for $25 \%{ }^{13} \mathrm{C}_{\alpha}$-enriched glycine at a $850 \mathrm{MHz}$ NMR spectrometer with $20 \mathrm{kHz}$ sample spinning. The observed line shapes reveal only minor differences between the various decoupling sequences. The performance of the $r \mathrm{CW}^{C}$ sequence follows the trends from Fig. 4, however, the basic element $r \mathrm{CW}^{A}$ performs remarkably well. In contrast, the increase in decoupling performance expected for the more elaborated $r \mathrm{CW}$ sequences are not observed. The reason for this is most likely insufficient performance of the refocusing pulses with the same phase as the CW decoupling. Despite this, we note that the $r \mathrm{CW}$ sequences perform as good as elaborately optimized (see Figure S1 in supplementary material ${ }^{36}$ ) TPPM, SPINAL, SW $f^{-T P P M}$, and $\mathrm{SW}_{f^{-}}$ SPINAL using the same average rf power. This may render the $r \mathrm{CW}$ decoupling the method of choice when taking into account improved robustness and ease of setup.

(a)
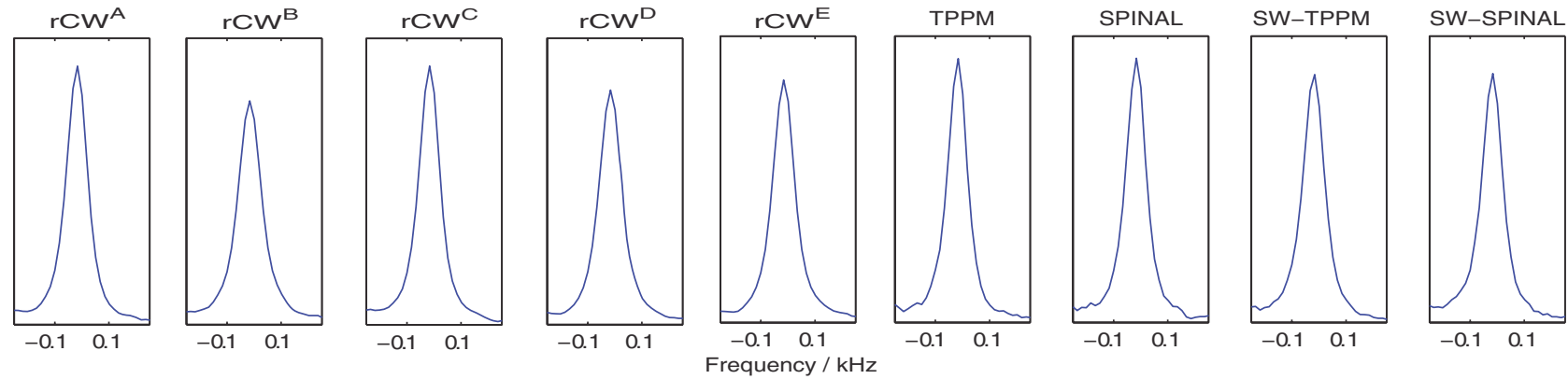

(b)
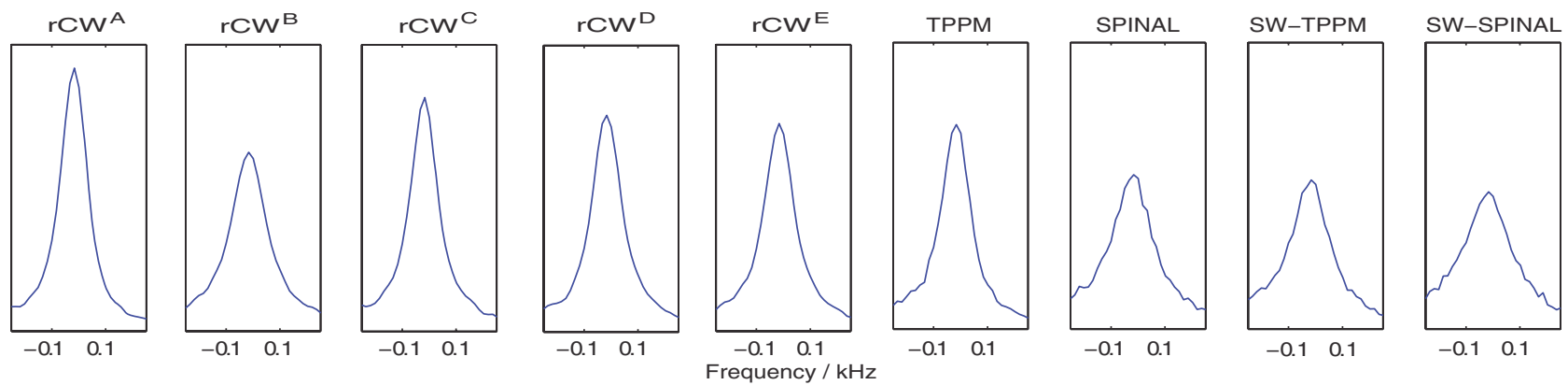

FIG. 7. Experimental comparison of ${ }^{13} \mathrm{C}_{\alpha}$ line shapes for $25 \%{ }^{13} \mathrm{C}_{\alpha}$-enriched glycine recorded at a $850 \mathrm{MHz}$ spectrometer, using $20 \mathrm{kHz}$ sample spinning and various decoupling sequences using (a) $93.7 \mathrm{kHz}$ (for the $r \mathrm{CW}$ sequences $90 \mathrm{kHz}$ for the $\mathrm{CW}$ irradiation and $222 \mathrm{kHz}$ for refocusing/purging pulses; for the TPPM and SPINAL type sequences $93.7 \mathrm{kHz}$ throughout) and (b) $70 \mathrm{kHz}$ (for the $r \mathrm{CW}$ sequences $70 \mathrm{kHz}$ for the $\mathrm{CW}$ irradiation and $222 \mathrm{kHz}$ for refocusing/purging pulses; for the TPPM and SPINAL type sequences $70 \mathrm{kHz}$ throughout) average rf power. The line shapes are obtained under optimized conditions by Fourier transformation of the free induction decay without apodization. 
Figure 7(b) shows the corresponding line shape plots obtained for decoupling sequences optimized to $70 \mathrm{kHz}$ average rf field strength (optimizations shown in the supplementary material), reflecting the interesting local optimum for the $r \mathrm{CW}$ decoupling sequences (cf. Fig. 6). In this case, we observe significant differences between the various decoupling sequences. The $r \mathrm{CW}$ sequences generally outperform the elaborately optimized SPINAL, $\mathrm{SW}_{f^{-}}$TPPM, and $\mathrm{SW}_{f^{-}}$ SPINAL decoupling sequences and the basic element $r \mathrm{CW}^{A}$ as well as the $r \mathrm{CW}^{C}$ sequence also outperform TPPM under these conditions. We note also here that the basic element $r \mathrm{CW}^{A}$ performs surprisingly well, which may be ascribed to a small value for $\omega_{y}^{(3)}$ (the non-refocused effective Hamiltonian, see Eq. (22)). This agrees well with the ordering $\omega_{y}^{(3)} \ll \omega_{z}^{(3)} \ll \omega_{x}^{(2)}$ as determined analytically for the $\mathrm{CH}$ spin system for $\omega_{r f}>3 \omega_{r}$ (cf. Fig. 1). As for the $93.7 \mathrm{kHz}$ analysis, the expected gain in decoupling efficiency by the more elaborate $r \mathrm{CW}$ sequences fails to appear, which we again attribute to insufficient performance of the refocusing pulses over long averaging periods or, potentially, non-addressed multiple-spin effects. We should also note that the earlier decoupling methods in the case of $70 \mathrm{kHz}$ rf field strength show different behavior mutually relative to what we observed in the case of $93.7 \mathrm{kHz}$ decoupling. This may be ascribed to differences in the dependencies of the sequences on the ratio between the rf field strength and the spinning speed-and may therefore alter upon changing the rf field strength in an additional optimization process. As so the analysis at $70 \mathrm{kHz}$ also serves to emphasize the difficulty in the optimization of previous methods, being alleviated to some extend using $r \mathrm{CW}$ decoupling.

\section{DISCUSSION}

The experimental results justify the relevance of our decoupling design principle and the overall usefulness of the $r \mathrm{CW}$ sequences. The performance is in general comparable to or marginally better than state-of-the-art decoupling sequences. The $r \mathrm{CW}$ sequences show robustness towards $\mathrm{rf}$ inhomogeneities and offsets, in this regard being clearly superior to commonly used decoupling sequences. However, as seen by comparing simulations (Fig. 4) and experimental (Fig. 7) performance, the most sophisticated $r \mathrm{CW}$ sequences $\left(r \mathrm{CW}^{B}, r \mathrm{CW}^{D}\right.$, and $\left.r \mathrm{CW}^{E}\right)$ do not display the expected improved performance as compared to the simpler $r \mathrm{CW}^{A}$ and $r \mathrm{CW}^{C}$ decoupling sequences. It has not been possible to ascribe this lack of improvement to specific parameters in the considered spin systems, rf inhomogeneity, or sample spinning instability. Accordingly, explanations may be sought in pulse imperfections such as finite rise/fall times and phase transients or multi-spin effects accumulating during the relatively slow averaging (long sequences) periods.

That pulse imperfections may play an important role may be rationalized considering that the difference between the experimentally best-functioning sequences $\left(r \mathrm{CW}^{A}\right.$ and $\left.r \mathrm{CW}^{C}\right)$ and the less well performing $\left(r \mathrm{CW}^{B}, r \mathrm{CW}^{D}\right.$, and $\left.r \mathrm{CW}^{E}\right)$ is the incorporation of refocusing pulses of same phase as the $\mathrm{CW}$ element in which they are incorporated. This observation could make the design strategy 2 (see Sec. III) particularly interesting, since it opens the possibility to avoid this kind of refocusing pulses. Moreover, it may be speculated that the improved removal of the homonuclear proton-proton interactions weakens the decoupling efficiency in terms of elimination of self-decoupling due to the large proton-proton dipolar couplings. ${ }^{41,54,55}$ Regarding the optimum for the delay between the refocusing pulses of around $100 \mu \mathrm{s}$, it is likely that a match with the rotational averaging is non-preferable, since the two averaging processes may interfere. Opposedly, the refocusing has to be frequent enough to be effective, putting an upper limit on the refocusing delay. The $r \mathrm{CW}$ sequences differ from most decoupling sequences by using averaging over time-periods longer than a rotor period, which makes the incorporation as decoupling during hetero-spin manipulations an interesting possibility.

Relative to TPPM, SPINAL, and to some extent also the swept variants of these (which are more stable towards phase and pulse length adjustment), the $r \mathrm{CW}$ sequences are easy to set up and optimize, and the sequences are robust towards rf inhomogeneity, resonance offsets, and chemical shielding anisotropy. Although some variants arguably are demanding with respect to peak rf power, the $r \mathrm{CW}$ decoupling sequences perform comparatively well when considering the average rf powers. Especially, the $r \mathrm{CW}^{C}$ sequence indicate a broad range of applications due to reduced demands to high-power refocusing pulses and its overall high decoupling efficiency. The simplicity of the $r \mathrm{CW}$ decoupling scheme may be attractive when used in advanced solid-state NMR experiments, e.g., in concert with recoupling pulse sequences on low- $\gamma$ rf channels.

\section{CONCLUSIONS}

A novel decoupling scheme and associated design principle have been presented, opening new ways for systematic development of decoupling sequences on the basis of simple two-spin and three-spin effective Hamiltonian analysis. The proposed $r \mathrm{CW}$ decoupling sequences show high decoupling efficiencies over a broad range of experimental parameters with performance comparable to or slightly better than stateof-the-art decoupling sequences such as TPPM, SPINAL, and swept versions of these. The $r \mathrm{CW}$ sequences are easy to set up and faster to optimize than TPPM and similar sequences. It is of particular interest to note that the $r \mathrm{CW}$ sequences reveal relatively good decoupling performance at low average decoupling rf field strengths and that the sequences are robust towards rf inhomogeneity, offsets, and anisotropic shielding of which the latter is particularly important in high-field applications.

\section{ACKNOWLEDGMENTS}

We acknowledge support from the Villum-Kahn Rasmussen Foundation, the Danish National Research Foundation, the FP7 BIONMR programme, and the Danish Center for Scientific Computing. We furthermore acknowledge the Netherlands Organization for Scientific Research (NWO) for 
their support of the solid-state NMR facility for advanced materials research.

${ }^{1}$ M. H. Levitt and R. Freeman, J. Magn. Reson. 33, 473 (1979).

${ }^{2}$ M. H. Levitt and R. Freeman, J. Magn. Reson. 43, 502 (1981).

${ }^{3}$ J. S. Waugh, J. Magn. Reson. 50, 30 (1982).

${ }^{4}$ Y. K. Lee, N. D. Kurur, M. Helmle, O. G. Johannessen, N. C. Nielsen, and M. Levitt, Chem. Phys. Lett. 242, 304 (1995).

${ }^{5}$ M. H. Levitt, J. Chem. Phys. 128, 052205 (2008).

${ }^{6}$ G. D. Paëpe, P. Hodgkinson, and L. Emsley, Chem. Phys. Lett. 376, 259 (2003).

${ }^{7}$ A. E. Bennett, C. M. Rienstra, M. Auger, K. V. Lakshmi, and R. G. Griffin, J. Chem. Phys. 103, 6951 (1995).

${ }^{8}$ B. M. Fung, A. K. Khitrin, and K. Ermolaev, J. Magn. Reson. 142, 97 (2000).

${ }^{9}$ A. Detken, E. H. Hardy, M. Ernst, and B. H. Meier, Chem. Phys. Lett. 356, 298 (2002).

${ }^{10}$ M. Weingarth, G. Bodenhausen, and P. Tekely, J. Magn. Reson. 199, 238 (2009)

${ }^{11}$ M. Eden and M. H. Levitt, J. Chem. Phys. 111, 1511 (1999).

${ }^{12}$ R. S. Thakur, N. D. Kurur, and P. K. Madhu, Chem. Phys. Lett. 426, 459 (2006).

${ }^{13}$ C. V. Chandran, P. K. Madhu, N. D. Kurur, and T. Bräuniger, Magn. Reson. Chem. 46, 943 (2008).

${ }^{14}$ C. V. Chandran and T. Bräuniger, J. Magn. Reson. 200, 226 (2009).

${ }^{15}$ C. Augustine and N. D. Kurur, J. Magn. Reson. 209, 156 (2011).

${ }^{16}$ S. Paul, N. D. Kurur, and P. K. Madhu, J. Magn. Reson. 207, 140 (2010).

${ }^{17}$ J. A. Stringer, C. E. Bronnimann, C. G. Mullen, D. H. Zhou, S. A. Stellfox, Y. Li, E. H. Williams, and C. M. Rienstra, J. Magn. Reson. 173, 40 (2005).

${ }^{18}$ F. D. Doty, J. Kulkarni, C. Turner, G. Entzminger, and A. Bielecki, J. Magn. Reson. 182, 239 (2006).

${ }^{19}$ P. L. Gor'kov, E. Y. Chekmenev, C. Li, M. Cotten, J. J. Buffy, N. J. Traaseth, G. Veglia, and W. W. Brey, J. Magn. Reson. 185, 77 (2007).

${ }^{20}$ B. Dillmann, K. Elbayed, H. Zeiger, M.-C. Weingertner, M. Piotto, and F. Engelke, J. Magn. Reson. 187, 10 (2007).

${ }^{21}$ R. W. Martin and K. W. Zilm, J. Magn. Reson. 165, 162 (2003).

${ }^{22}$ P. C. A. van der Wel, J. R. Lewandowski, and R. G. Griffin, J. Am. Chem. Soc. 129, 5117 (2007).

${ }^{23}$ L. Sperling, A. Nieuwkoop, A. Lipton, D. Berthold, and C. Rienstra, J. Biomol. NMR 46, 149 (2010).

${ }^{24}$ S. K. Vasa, H. Janssen, E. R. H. V. Eck, and A. P. M. Kentgens, Phys. Chem. Chem. Phys. 13, 104 (2011)

${ }^{25}$ G. D. Paëpe, N. Giraud, A. Lesage, P. Hodgkinson, A. Böckmann, and L. Emsley, J. Am. Chem. Soc. 125, 13938 (2003).

${ }^{26}$ M. Ernst, J. Magn. Reson. 162, 1 (2003).
${ }^{27}$ C. Filip, S. Hafner, I. Schnell, D. E. Demco, and H. W. Spiess, J. Chem. Phys. 110, 423 (1999).

${ }^{28}$ J. R. Lewandowski, J. Sein, H. J. Sass, S. Grzesiek, M. Blackledge, and L. Emsley, J. Am. Chem. Soc. 132, 8252 (2010).

${ }^{29}$ G. D. Paëpe, B. Elena, and L. Emsley, J. Phys. Chem. 121, 3165 (2004).

${ }^{30}$ A. McDermott, F. Creuzet, A. Kolbert, and R. Griffin, J. Magn. Reson. 98, 408 (1992)

${ }^{31}$ U. Akbey, S. Lange, W. Trent Franks, R. Linser, K. Rehbein, A. Diehl, B.-J. van Rossum, B. Reif, and H. Oschkinat, J. Biomol. NMR 46, 67 (2010).

${ }^{32}$ U. Haeberlen and J. S. Waugh, Phys. Rev. 175, 453 (1968).

${ }^{33}$ M. Hohwy and N. C. Nielsen, J. Chem. Phys. 109, 3780 (1998).

${ }^{34}$ T. S. Untidt and N. C. Nielsen, Phys. Rev. E 65, 021108 (2002).

${ }^{35}$ M. Ernst, A. Samoson, and B. H. Meier, J. Chem. Phys. 123, 64102 (2005).

${ }^{36}$ See supplementary material at http://dx.doi.org/10.1063/1.4768953 for the solution to the triple integral and the $70 \mathrm{kHz}$ rf-amplitude optimizations.

${ }^{37}$ G. Peano, Math. Ann. 32, 450-456 (1888).

${ }^{38}$ H. F. Baker, Proc. London Math. Soc. s2-2, 293 (1905).

${ }^{39}$ M. Baake and U. Schlägel, Proc. Steklov Inst. Math. 275, 155 (2011).

${ }^{40}$ P. Caravatti, G. Bodenhausen, and R. R. Ernst, J. Magn. Reson. 55, 88 (1983).

${ }^{41}$ M. Mehring, High Resolution NMR in Solids (Springer-Verlag, New York, 1976).

${ }^{42}$ B. Berglund and R. W. Vaughan, J. Chem. Phys. 73, 2037 (1980)

${ }^{43}$ M. Hohwy and N. C. Nielsen, J. Phys. Chem. 106, 7571 (1997).

${ }^{44}$ M. Hohwy, P. V. Bower, H. J. Jakobsen, and N. C. Nielsen, Chem. Phys. Lett. 273, 297 (1997).

${ }^{45}$ M. Bak, J. T. Rasmussen, and N. C. Nielsen, J. Magn. Reson. 147, 296 (2000).

${ }^{46}$ T. Vosegaard, A. Malmendal, and N. C. Nielsen, Monatsch. Chem. 133, 1555 (2002).

${ }^{47}$ Z. Tošner, T. Vosegaard, C. Kehlet, N. Khaneja, S. J. Glaser, and N. C. Nielsen, J. Magn. Reson. 197, 120 (2009).

${ }^{48}$ M. Bak, R. Schultz, T. Vosegaard, and N. C. Nielsen, J. Magn. Reson. 154, 28 (2002).

${ }^{49}$ M. Bak and N. C. Nielsen, J. Magn. Reson. 125, 132 (1997).

${ }^{50}$ Wolfram Research, Inc., Mathematica Edition: Version 8.0 (Wolfram Research, Inc., Champaign, IL, 2010).

${ }^{51}$ J. D. van Beek, J. Magn. Reson. 187, 19 (2007).

${ }^{52}$ M. Kotecha, N. P. Wickramasinghe, and Y. Ishii, Magn. Reson. Chem. 45, S221 (2007).

${ }^{53}$ I. Scholz, P. Hodgkinson, B. H. Meier, and M. Ernst, J. Chem. Phys. 130, 114510 (2009).

${ }^{54}$ A. Abragam and J. Winter, C. R. Hebd. Seances Acad. Sci. 249, 1633 (1959).

${ }^{55}$ A. Abragam, Principles of Nuclear Magnetism (Clarendon, 1961).

${ }^{56}$ M. Leskes, N. D. Kurur, P. K. Madhu, R. S. Thakur, S. Vega, J. Chem. Phys. 127, 024501 (2007). 